\title{
CARTOGRAPHIE DES CULTURES DANS LE PÉRIMĖTRE DU LOUKKOS (MAROC) : APPORT DE LA TÉLÉDÉTECTION RADAR ET OPTIQUE
}

\author{
Siham Acharki ${ }^{1}$, Mina Amharref ${ }^{1}$, Pierre-Louis Frison², Abdes Samed Bernoussi ${ }^{1}$ \\ 1 Équipe GAT, Faculté des Sciences et Techniques de Tanger, \\ Université Abdelmalek Essaadi, Tanger, Maroc, sacharkis@gmail.com \\ 2 LaSTIG/MATIS, Université Gustave Eiffel, IGN, \\ 5 Bd Descartes, Champs sur Marne, 77455 Marne la Vallée CEDEX 2, France, pierre-louis.frison@u-pem.fr
}

\begin{abstract}
Résumé
Dans cet article, nous analysons la possibilité d'amélioration de la classification des cultures dans un périmètre irrigué du nord du Maroc en se basant sur la combinaison des données multi-temporelles de deux satellites (Sentinel-1 et Sentinel2) avec l'inclusion de neuf indices. Le périmètre concerné (Loukkos), en plus de sa position stratégique, se caractérise par un climat méditerranéen avec une forte valeur écologique. II présente une intense activité agricole avec une grande diversité des cultures dont le fonctionnement pourrait être affecté par le changement climatique. Afin de quantifier les besoins en eau, nous avons utilisé les séries d'images satellitaires acquises pour la période du 09/2017 au 08/2018. Les cartes produites pour trois niveaux de classification illustrent notre approche. L'étude a montré que les 10 canaux optiques, à 10 et $20 \mathrm{~m}$ de résolution spatiale, des données acquises par Sentinel-2 permettent d'obtenir de bonnes performances, avec un indice de kappa $>85 \%$ pour toutes les sous-classes et une précision globale $>86 \%$. Ces performances sont supérieures à celles obtenues avec des données radar acquises par Sentinel-1, avec des écarts de F-score inférieurs de $9 \%$ en moyenne, et pouvant aller jusqu'à $29 \%$ (sur le chêne-liège/Niveau SSC). Ni l'ajout d'indices radiométriques optiques, ni la combinaison des données optiques et radar n'apportent d'amélioration significative aux performances obtenues avec les données Sentinel-2. Afin d'exploiter les données obtenues, les travaux à venir se focaliseraient sur l'étude des profils temporels de chaque type de culture.
\end{abstract}

Mots-clés : Sentinel-1, Sentinel-2, Classification supervisée, Forêt aléatoire, Cultures, Loukkos

\begin{abstract}
In this article, we analyze the possibility to improve the classification of crops in an irrigated area of northern Morocco based on the combination of the multi-temporal data of two satellites (Sentinel-1 and Sentinel-2) with the inclusion of nine indices. The area concerned (Loukkos), in addition to its strategic position, is characterized by a Mediterranean climate with a strong ecological value. It presents an intense agricultural activity with a great diversity of cultures whose functioning could be affected by climate change. To quantify water requirements, we used the series of satellite images acquired during 09/2017 to 08/2018. Maps produced for three classification levels illustrate our approach. The study showed that the 10 optical channels, at 10 and $20 \mathrm{~m}$ spatial resolution, of the data acquired by Sentinel-2 provide good performance, with a kappa index $>85 \%$ for all (sub)classes and an overall accuracy $>86 \%$. These performances are superior to those obtained with radar data acquired by Sentinel-1, with F-score deviations of $9 \%$ lower on average, and up to $29 \%$ (on cork oak/SSC level). Neither the addition of optical radiometric indices, nor the optical and radar combination data provide a significant improvement in performance obtained with Sentinel-2 data. In order to exploit the data obtained, future work would focus on studying the temporal profiles of each type of crop.
\end{abstract}

Keywords: Sentinel-1, Sentinel-2, Supervised classification, Random Forest, Crop, Loukkos

\section{Introduction}

Les cartes d'utilisation des terres constituent des données d'entrée importantes pour les modèles hydrologiques. Elles permettent de quantifier les besoins en eau dans un contexte de fortes variations climatiques. La télédétection s'est révélée être un outil utile et fréquemment utilisé dans le suivi et la cartographie d'utilisation des terres notamment des cultures (Sonobe et al., 2017 ; Veloso et al., 2017 ; Tricht et al., 2018). En outre, la combinaison de données satellitaires optiques et radar peut fournir des informations importantes sur les types de culture et permet d'estimer leurs paramètres biophysiques (Veloso et al., 2017 ; Tricht et al., 2018). Des études récentes ont montré que la combinaison de ces différents capteurs peut considérablement augmenter la précision de la classification. Tricht et al.
(2018) ont utilisé des images Sentinel-1 et Sentinel-2 afin de cartographier les cultures en Belgique. Une classification supervisée par forêt aléatoire optimisée a été réalisée. Cependant, le nombre de classes était limité ( 8 types de culture). Les résultats révélaient que la précision maximale est de $82 \%$ avec un coefficient kappa de 0,77 . Sonobe et al. (2017) combinaient cinq images Sentinel-1 et une image Sentinel-2 mais en comparant quatre approches de classification à Hokkaido, Japon. Eux aussi se sont limités à six types de cultures avec une précision de $96,8 \%$. Ils suggèrent l'utilisation plus étendue de la combinaison des données des deux satellites en utilisant l'approche machine d'apprentissage extrême basée sur le noyau (KELM). Steinhausen et al. (2018) ont identifié treize classes d'occupation des sols dans le bassin de Chennai en Inde à l'aide de la série temporelle Sentinel-1 et Sentinel-2. 
Cette étude montre la pertinence de la combinaison pour améliorer la précision globale de la cartographie de différentes classes, avec une amélioration de 5,7\% comparée à l'utilisation de Sentinel-2 uniquement.

Au Maroc, très peu d'études (Simonneaux et al., 2008; Hadria et al., 2009 ; Höpfner et Scherer, 2011 ; Mohajane et al., 2018) ont traité l'utilisation séparée ou combinée de données multi-temporelles optique/radar, et rares sont celles (Ouzemou et al., 2018 ; Htitiou et al., 2019) qui l'ont fait pour l'identification des types de cultures. A notre connaissance aucune n'a fait l'étude optique/radar à plusieurs niveaux de classification. Dans cet article, nous étudions la possibilité de combiner les données Sentinel-1 et Sentinel-2 afin d'améliorer la cartographie des cultures dans le périmètre irrigué Loukkos (Nord du Maroc) suivant une nomenclature hiérarchique à trois niveaux de classification.

Les questions auxquelles nous tentons de répondre sont les suivantes :

- La combinaison de l'imagerie radar et optique fournit-elle vraiment une amélioration de la précision pour l'identification du type de culture? Et de combien?

- Quels sont les types de culture les mieux détectés par le radar et/ou optique et/ou indices?

- Y a-t-il une cohérence en termes de superficie entre les trois niveaux de classification?

Le papier est structuré comme suit : Dans la section suivante nous présentons des informations sur la région d'étude. Dans la section 3 nous décrivons la méthodologie suivie. Dans la section 4 nous interprétons et discutons les résultats obtenus. Enfin, les principales conclusions sont données dans la section 5

\section{Zone d'étude}

Notre région d'étude est située principalement dans le périmètre irrigué Loukkos-Nord du Maroc (figure 1). Elle couvre une superficie totale de $1691 \mathrm{~km}^{2}$ soit $66,60 \%$ de la superficie totale dudit périmètre. Sa topographie est un peu plus accidentée que celle des périmètres avoisinants (tels que Gharb) et la majeure partie de son altitude est inférieure à $100 \mathrm{~m}$. La région se caractérise par un climat méditerranéen avec une température moyenne annuelle de $18,61{ }^{\circ} \mathrm{C}$ et des précipitations annuelles de $632 \mathrm{~mm}$.

De plus, elle présente une intense activité agricole avec une diversité de cultures. Parmi ces cultures, on cite : céréales et fourrages (avoine, blé, maïs et orge), maraîchage (ail, aubergine, carotte, concombre, courgette, choux, fève, haricot vert, laitue, melon, pomme de terre, pastèque, poivron, piment, oignon et tomate), fruits rouges (fraise, framboise, myrtille et mûrier), oléagineux (arachide), cultures industrielles (betterave et canne à sucre), arboriculture (avocatier, agrumes, kaki, olivier et prunier) et rizière. Dans cet article, nous nous limitons à l'étude de treize types de cultures. Le paysage associé à ces cultures est varié en termes de structure de végétation (érectophile, hauteur de végétation, ...), géométrie de couvert (arboré, densité, plantation en rang, ...) comme l'illustre la figure 2. Leur cycle phénologique est présenté dans la figure 3.

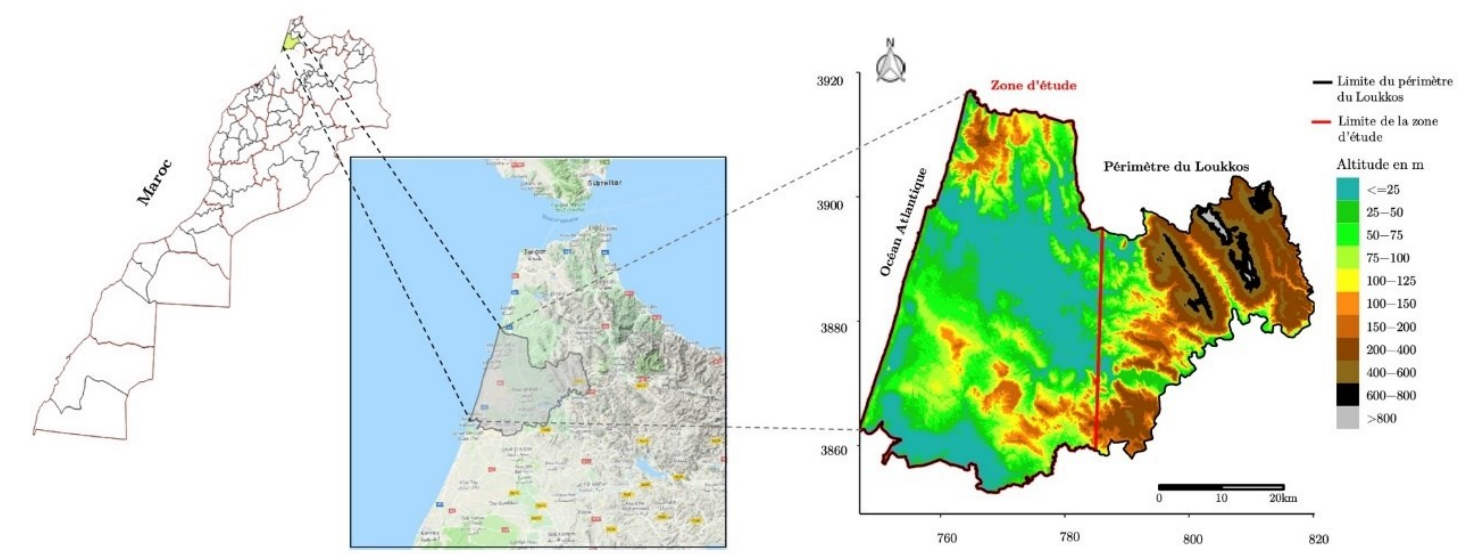

Figure 1: Localisation géographique et carte d'élévation (SRTM) de la région d'étude (Périmètre du Loukkos, Maroc) selon le système de projection UTM29.

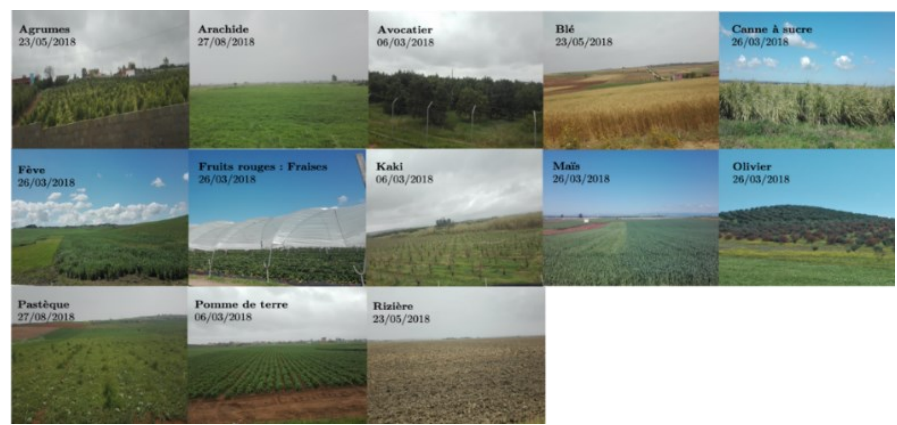

Figure 2: Différents types de cultures. Périmètre du Loukkos (Maroc). 


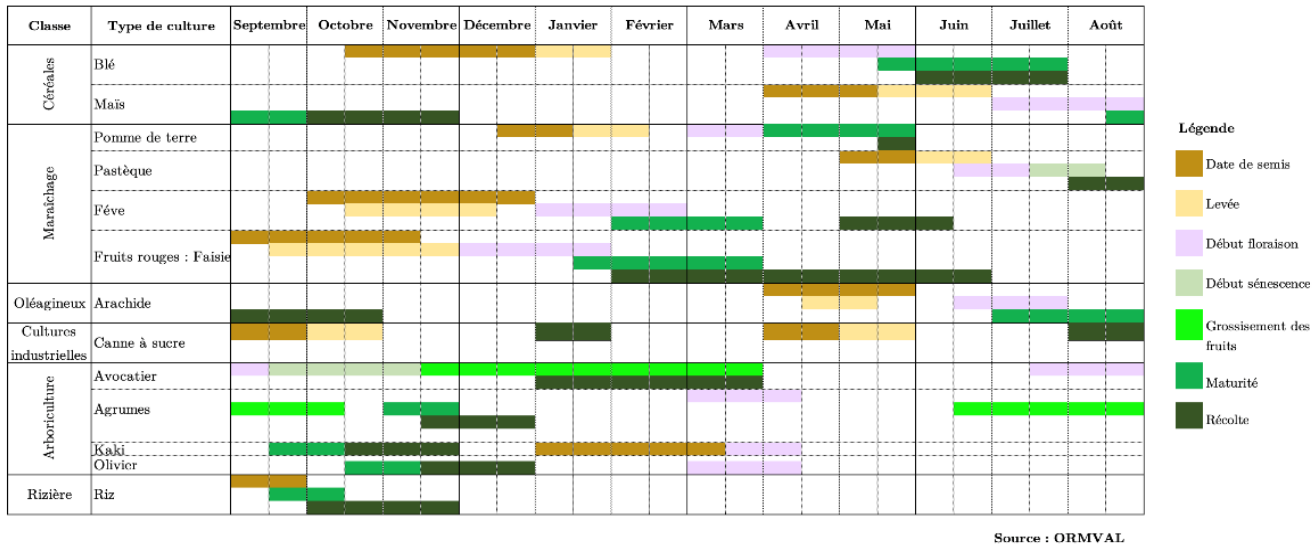

Figure 3: Calendrier cultural pour certaines cultures clés du périmètre du Loukkos (Maroc).

\section{Méthodologie}

Les étapes de pré-traitement et de traitement suivies dans cet article sont résumées dans la figure 4.

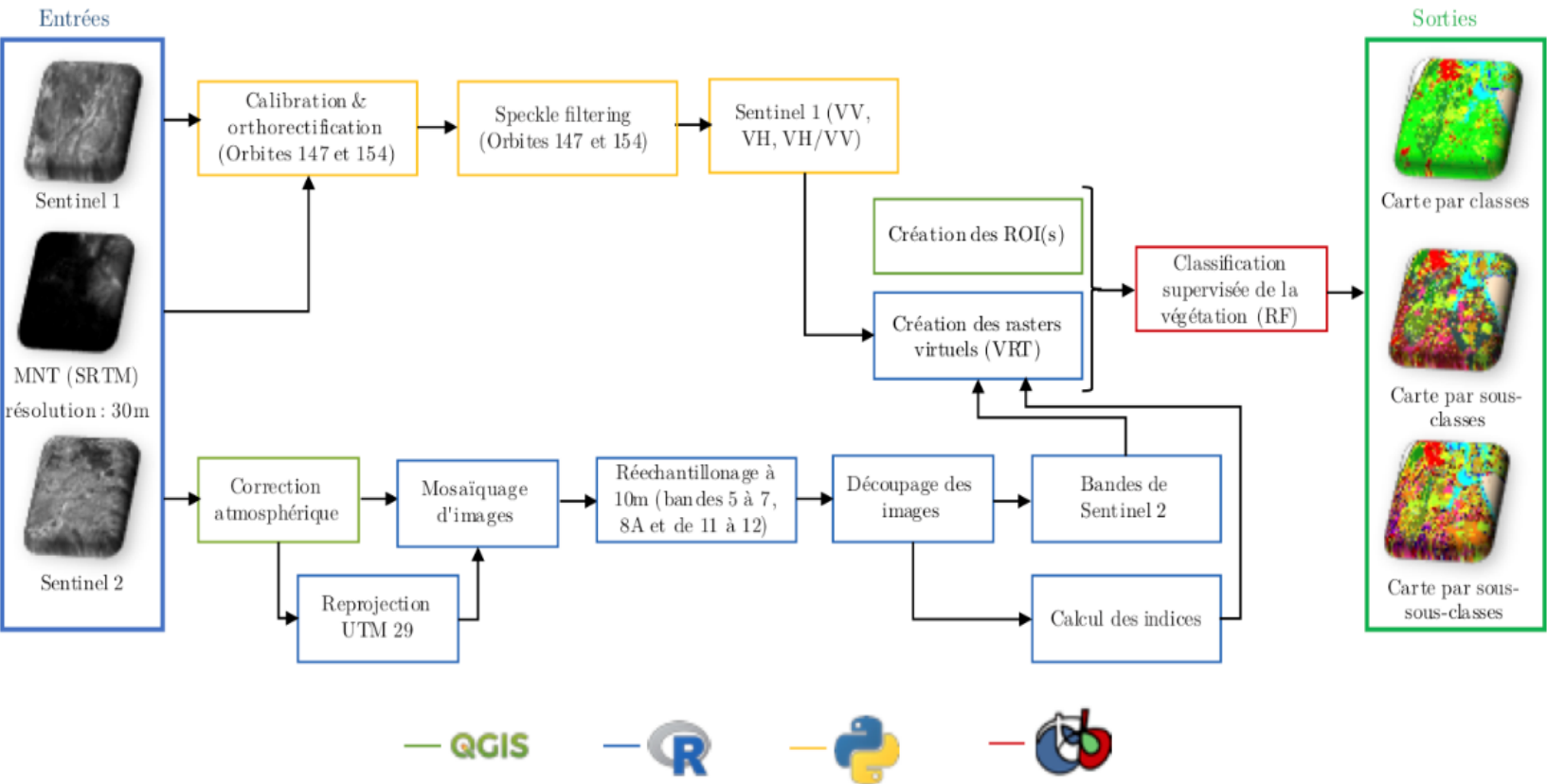

Figure 4: Les différentes étapes de traitement des images satellitaires

\subsection{Acquisition des images}

Les images radar et optique ont été acquises via la plateforme Scihub Copernicus (Copernicus, 2018) pour la période du 1er septembre 2017 au 31 août 2018 sur la région d'étude (figure 1). Les images Sentinel-1 comprennent des données GRD de niveau 1 en mode interférométrique large (IW). Dans cet article, nous exploitons la série chronologique de 43 images montantes (orbite 147) et 31 images descendantes (orbite 154) soit un total de 74 images. Les

images Sentinel-2 A et B sont de niveau 1C. En raison de la présence des nuages, nous avons obtenu 24 images (soit 2 images non mosaïquées par date et avec un pourcentage de tolérance de nuage de $0 \%$ ). La création d'une mosaïque a été effectuée puisque notre région d'étude est couverte par deux tuiles de $100 \times 100 \mathrm{~km}^{2}$. La figure 5 présente les principales dates d'acquisition des images issues des deux satellites.

\subsection{Prétraitement Sentinel-1 et Sentinel-2}

Le traitement des images Sentinel-1 a été effectué via des scripts python basés sur le logiciel Orfeo Toolbox (OTB, 2018). Ce traitement est réalisé à travers différentes phases comme déjà décrit dans le chapitre de (Frison et Lardeux, 2018). La calibration radiométrique consiste à convertir les valeurs numériques afin d'obtenir le 
coefficient de rétrodiffusion $\left(\sigma^{o}\right)$. Ensuite, pour corriger les distorsions géométriques dues aux changements de position et d'attitude du satellite, des corrections de terrain ont été appliquées à l'aide du modèle d'élévation numérique (SRTM). Ce modèle MNT a été téléchargé via la plateforme Earth Explorer (USGS, 2018) et a une résolution spatiale de $30 \mathrm{~m}$. Finalement, un filtre multi- temporel a été généré afin de réduire les effets de chatoiement. Cette méthode de filtrage est particulièrement adaptée aux séries temporelles constituées d'un grand nombre d'images, telles que celles acquises par Sentinel-1 (Frison et Lardeux, 2018). Elle permet de réduire le speckle tout en préservant la résolution spatiale des images (Baghdadi et Zribi, 2017).

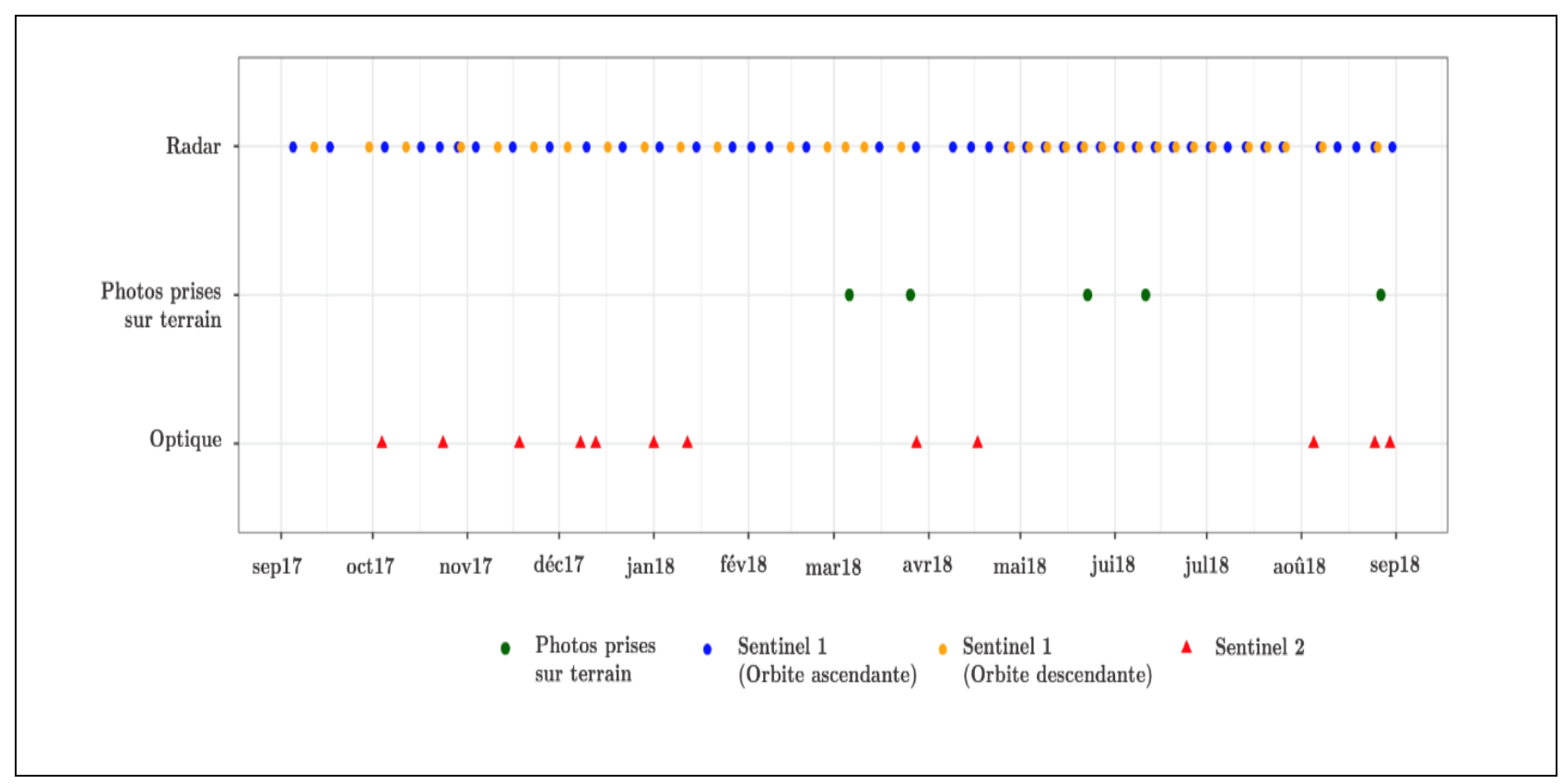

Figure 5: Dates d'acquisition des images satellitaires. Périmètre du Loukkos (Maroc).

Le processus de traitement des images Sentinel-2 a comporté cinq étapes. La correction atmosphérique consiste à convertir d'une part le nombre numérique DN en radiance TOA et d'autre part la réflectance TOA en valeurs de réflectance de surface en utilisant l'algorithme de soustraction d'objet sombre (DSO1). Ce processus a été fait à l'aide du plugin SCP développé par L. Congedo (Congedo, 2016). Nous avons appliqué un processus de reprojection aux images ayant un système de projection UTM30 et un système de WGS 84 . En outre, les bandes acquises à une résolution de $20 \mathrm{~m}(5,6,7,8 \mathrm{a}, 11$ et 12) ont été ré-échantillonnées à $10 \mathrm{~m}$ en utilisant la méthode du plus proche voisin. Une fois les bandes réechantillonnées produites, elles ont été découpées en utilisant les limites de la région d'étude. À signaler que les quatre dernières étapes ont été implémentées sous le logiciel R (R Development Core Team, 2017).

\subsection{Indices}

Neuf indices radiométriques ont été générés à partir de données multispectrales Sentinel-2. Les formules de ces indices sont mentionnées dans le tableau 1.
L'indice d'eau par différence normalisée (NDWI : (Gao, 1996), permet de détecter l'eau ou les zones bien arrosées/ irriguées. L'indice de zones bâties par différence normalisée (NDBI : (Zha et al., 2003)), est utilisé pour indiquer la densité de construction d'une zone. La valeur de NDBI plus proche de 1 indique une densité élevée de terrains bâtis. L'indice du sol nu (BSI : (Rikimaru et al., 2002)), permet de quantifier la composition minérale du sol et est utilisé pour améliorer la détection de végétation. L'indice de végétation par différence normalisée (NDVI : (Rouse et al., 1974)), est l'indice le plus utilisé en télédétection pour analyser de la végétation verte (Veloso et al., 2017). Cependant, il est sensible aux perturbations atmosphériques et signaux de la couverture du sol au-dessous de la végétation (Huete et al., 2002). L'indice de végétation avancé (AVI : (Rikimaru et al., 2002)), est utilisé pour surveiller les variations des cultures et des forêts. Toutefois, il sensible à la densité de végétation par rapport à NDVI. L'indice de végétation amélioré (EVI : (Huete et al., 2002)), est une amélioration du NDVI en ajoutant un facteur d'ajustement du sol et deux coefficients de correction pour la diffusion des aérosols atmosphériques (Jensen, 2005). 
Tableau 1: Caractéristiques des indices utilisés pour les images Sentinel-2

$[R B, R G, R R$, RNIR, RSWIR1et RVRE sont respectivement les valeurs de réflectance dans les bandes visible bleue, verte, rouge, proche infrarouge, infrarouge moyen et bande $5 V R E]$

\begin{tabular}{|c|c|c|c|}
\hline Catégorie & Indice & Formule & Référence \\
\hline Eau & NDWI & $N D W I=\frac{R_{G}-R_{N I R}}{R_{c}+R_{N I I}}$ & $(\mathrm{Gao}, 1996)$ \\
\hline Urbain & NDBI & $\mathrm{NDBI}=\frac{R_{\mathrm{SWIR} 1}-R_{N I R}}{R_{\mathrm{SWIR} 1}+R_{\text {NIR }}}$ & (Zha et al., 2003) \\
\hline Sol & BSI & $\mathrm{BSI}=\frac{\left(R_{\mathrm{SWIR} 1}+R_{R}\right)-\left(R_{\mathrm{NIR}}-R_{B}\right)}{\left(R_{\text {SWIR } 1}+R_{R}\right)+\left(R_{\mathrm{NIR}}-R_{B}\right)}$ & (Rikimaru et al., 2002) \\
\hline \multirow[t]{6}{*}{ Végétation } & AVI & $\mathrm{AVI}=\sqrt[3]{R_{N I R} \times\left(1-R_{R}\right) \times\left(R_{\mathrm{NIR}}-R\right)}$ & (Rikimaru et al., 2002) \\
\hline & NDVI & $\mathrm{NDVI}=\frac{R_{N I R}-R_{R}}{R_{N I R}+R_{R}}$ & (Rouse et al., 1974) \\
\hline & OSAVI & OSAVI $=\frac{R_{N I R}-R_{R}}{R_{N I R}+R_{R}+0,16}$ & (Rondeaux et al., 1996) \\
\hline & $\mathrm{EVI}$ & $\mathrm{EVI}=2,5 \times \frac{R_{N I R}-R_{R}}{R_{N I R}+8 \times R_{R}-7,5 \times R_{R}+1}$ & (Huete et al., 2002) \\
\hline & LAISAVI & LAISAVI $=0,1663 \times \mathrm{e}^{4,2731 \mathrm{MSAVI}}$ & (Haboudane et al., 2004) \\
\hline & PSRI & PSRI $=\frac{R_{R}-R_{B}}{R_{V B E}}$ & (Merzlyaka et al., 1999) \\
\hline
\end{tabular}

L'indice de végétation ajusté au sol optimisé (OSAVI, Rondeaux et al., 1996) est utilisé dans les zones de végétation relativement clairsemée où le sol est visible à travers la canopée (Rondeaux et al., 1996). L'indice de surfaces foliaires (LAISAVI : (Haboudane et al., 2004)), permet d'estimer la couverture foliaire et d'analyser la production et le rendement des cultures (Haboudane et al., 2004). L'indice de sénescence des plantes (PSRI: (Merzlyaka et al., 1999)), permet de suivre la santé de la végétation et détecter le stress physiologique des plantes. Une augmentation du PSRI indique une augmentation du stress de la canopée. La distinction des différents types de végétation et l'extraction des caractéristiques/ paramètres phénologiques peut se faire grâce à la combinaison multi-temporelle des indices.

\subsection{Empilement des images/Création des rasters virtuels}

La combinaison des différentes bandes (optiques et/ou indices et/ou radar) a été réalisée. Les bandes 1, 9 et 10 de Sentinel-2 ont été exclues en raison de leur sensibilité aux aérosols et nuages ainsi que de leur résolution spatiale $(60 \mathrm{~m})$ (ESA, 2015). En résumé, les couches d'images résultantes ont été empilées en une image de plusieurs bandes, pour produire les jeux de données suivants :

- S1: Images Sentinel-1: VH, VV et leur rapport VH/VV (au total 222 bandes $=3$ polarisations * 74 acquisitions). - S2 : Images Sentinel-2: Bandes de réflectance 2 à $8 \mathrm{~A}$ et 11 à 12 (au total 120 bandes $=10$ canaux * 12 acquisitions).

S2Id : Images Sentinel-2 et les neuf indices (au total 228 bandes $=(10$ canaux +9 indices $) * 12$ acquisitions $)$. S1S2: Images Sentinel-1 et Sentinel-2 (au total 342 bandes $=120+222$ )

- S1S2Id: Images Sentinel-1, Sentinel-2 et indices (au total 450 bandes $=222+228$ ).

\subsection{Création des régions d'intérêt}

Nous avons créé des données d'entraînement (Régions d'intérêt ROI) d'une part sur la base des photos géolocalisées issues d'enquêtes de terrain menées durant les mois de mars à août 2018. D'autre part, par photo-interprétation d'images multi-temporelles haute résolution dans GoogleEarth. Nous avons considéré une classification hiérarchique à trois niveaux. Le premier niveau concerne les classes (C) qui sont largement utilisés dans la littérature. Le deuxième niveau nommé sous-classe (SC), permet de distinguer entre cinq grandes classes de cultures et trois classes de forêts. Dans le troisième niveau sous-sous-classe (SSC), les grandes classes de cultures ont été subdivisées en différents types de cultures afin de voir plus finement leur potentiel de discrimination. Ceci nous a permis de retenir huit classes, quatorze sous-classes et vingt-et-une soussous-classes. Le choix de ces dernières, repose sur la disponibilité des classes rencontrées lors des enquêtes sur terrain. Un diagramme en boîte à moustaches représentant la moyenne, le quartiles et déciles extrêmes de chacune de ces sous-classes, basée sur les polygones d'intérêts utilisés, est donné en figure 6 . La dénomination des sous-classes est donnée en tableau 2.

Sur la base de ces données, un total de 688 polygones soit 756817 pixels couvraient la zone en $2017 / 2018$. La figure 7 montre la répartition spatiale de ces polygones d'entraînement. Nos jeux de données utilisés ont été répartis au hasard en deux parties : $70 \%$ du jeu de données ont été affectés à l'initialisation du modèle de classification (RF), tandis que $30 \%$ de données restantes ont été utilisés pour la validation et l'évaluation des performances du modèle. La répartition a été effectuée au niveau du polygone. 


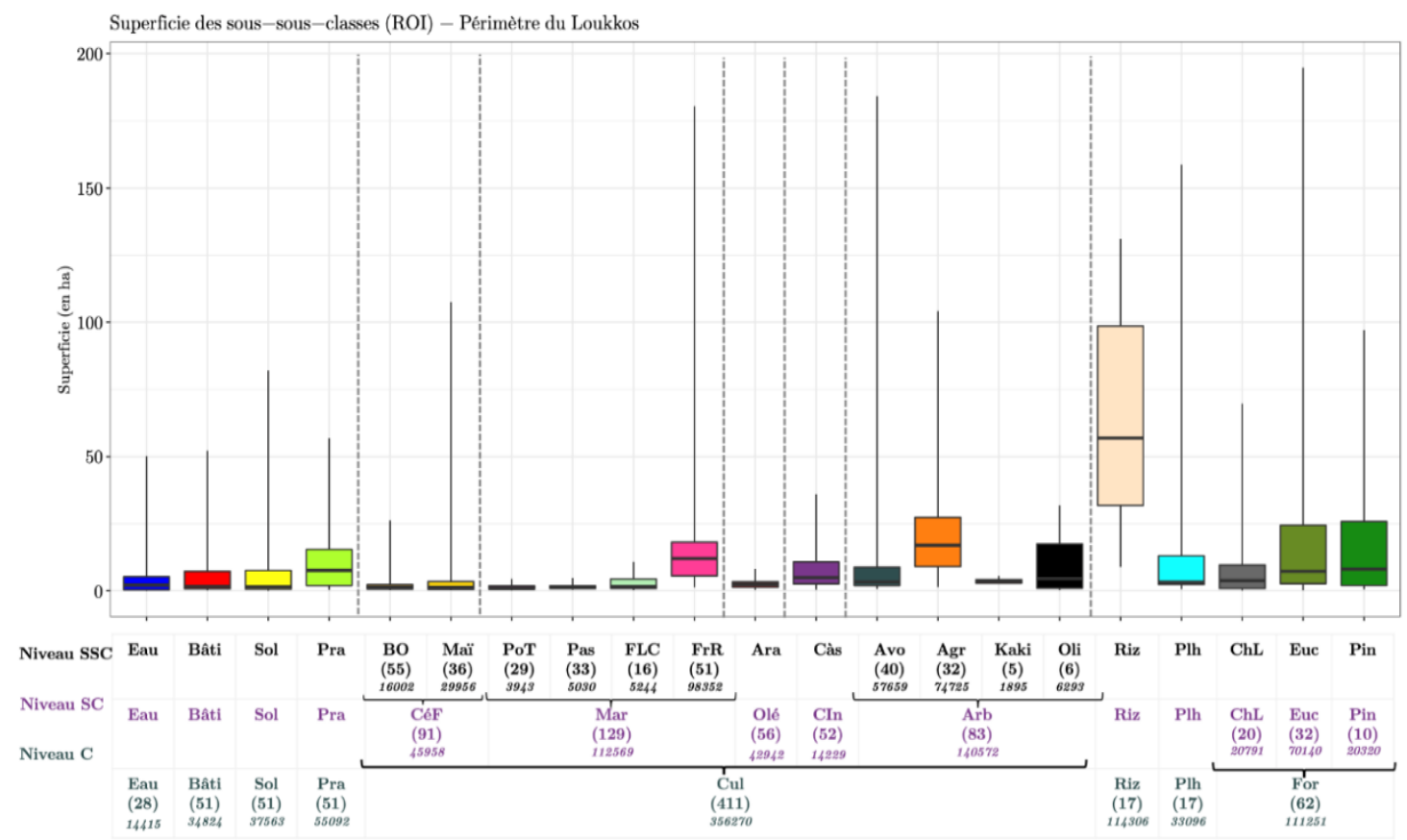

Figure 6: Superficie des ROls selon les classes du niveau sous-sous-classe. Périmètre du Loukkos (Maroc). Le nombre de polygones et le nombre de pixels par (sous-)classes sont donnés entre parenthèse et en italique respectivement.

Tableau 2: Dénomination des sous-classes.

\begin{tabular}{|l|l|}
\hline \multicolumn{1}{|c|}{ Niveau } & \multicolumn{1}{c|}{ sous-classe } \\
\hline Classe (C) & $\begin{array}{l}\text { Eau ; Bâtiments \& infrastructures ; Sol } \\
\text { nu ; Prairie ; Cultures ; Rizière ; Plantes } \\
\text { hydrophiles ; Forêt. }\end{array}$ \\
\hline $\begin{array}{l}\text { Sous- } \\
\text { classe } \\
\text { (SC) }\end{array}$ & $\begin{array}{l}\text { Eau ; Bâtiments \& infrastructures ; Sol } \\
\text { nu ; Prairie ; Céréales et fourrages ; } \\
\text { Maraîchage ; Oléagineux ; Cultures } \\
\text { industrielles ; Arboriculture ; Rizière ; } \\
\text { Plantes hydrophiles ; Chêne-liège ; } \\
\text { Eucalyptus ; Pin. }\end{array}$ \\
\hline $\begin{array}{l}\text { Sous- } \\
\text { sous- } \\
\text { classe } \\
\text { (SSC) }\end{array}$ & $\begin{array}{l}\text { Eau ; Bâtiments \& infrastructures ; Sol } \\
\text { nu ; Prairie ; Blé \& Orge ; Maïs ; Pomme } \\
\text { de terre ; Pastèque ; Fève, Laitue \& } \\
\text { Choux; Fruits rouges ; Arachide ; } \\
\text { Canne à sucre ; Avocatier ; Agrumes ; } \\
\text { Kaki, Olivier ; Rizière ; Plantes } \\
\text { hydrophiles ; Chêne-liège ; Eucalyptus ; } \\
\text { Pin. }\end{array}$ \\
\hline
\end{tabular}

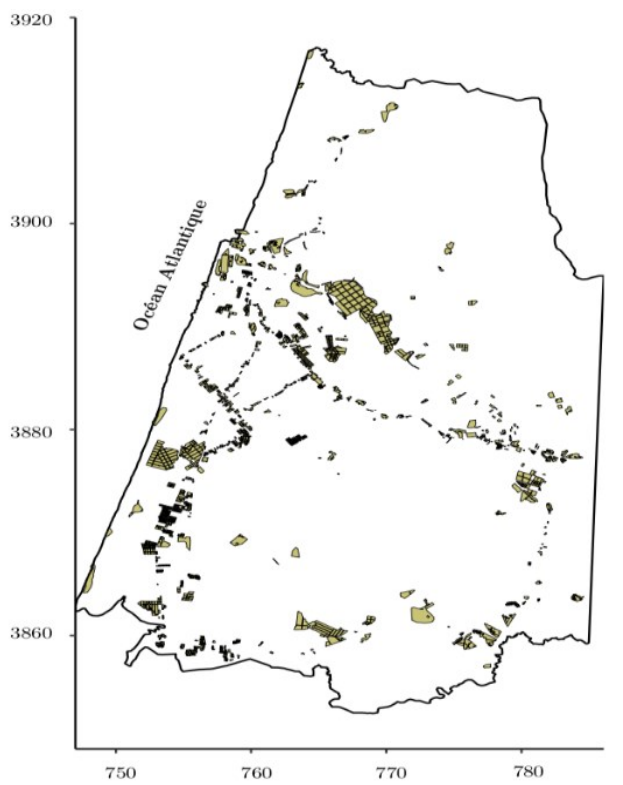

Figure 7 : Répartition des polygones des données d'entraînement (ROI). Périmètre du Loukkos (Maroc). 


\subsection{Classification et évaluation de la précision}

La classification des cinq jeux de données a été réalisée à l'aide d'une classification supervisée par forêts aléatoires (RF) mise en œuvre dans le logiciel Orfeo Toolbox (OTB, 2018). II s'agit d'un algorithme nonparamétrique (Breiman, 2001), qui consiste à combiner l'approche d'arbres de décision et celui d'agrégation.

En outre, il est capable de traiter des données de télédétection de grandes dimensions (Tricht et al., 2018; Sonobe et al., 2017). Il est également plus robuste sur le plan informatique, d'autres avantages et limites ont été mentionnés dans l'article de (Belgiu et Dragut, 2016). Ce classificateur nécessite le réglage de deux principaux paramètre, ntree (nombre d'arbres à cultiver dans la série) et Mtry (nombre d'entités utilisées dans chaque division). Dans cet article, nous avons utilisé les valeurs par défaut et défini ntree à 25 arbres et Mtry à 25 . Notons que nous avons fait 10 itérations de classificateur de forêt aléatoire pour chaque niveau de classification (ex. classe, sous-classes, ...) et chaque scénario (ex. S1, S2, ....). Ensuite, nous avons calculé leur moyenne harmonique.

Chaque classification produite a été évaluée à l'aide de la précision (précision du producteur) [Eq. 1], de rappel (précision de l'utilisateur) [Eq. 2], de F-Score [Eq. 3, (Rijsbergen, 1979)], de la précision globale [Eq. 4] et du coefficient Kappa [Eq. 5, (Foody, 1992)]. La précision est le nombre d'éléments correctement classés par rapport au nombre total d'éléments de cette classe au sein de la classification et se réfère aux erreurs d'omission. Le rappel, quant à lui, concerne les erreurs de commission et indique le nombre d'éléments correctement classés par rapport au nombre total d'éléments de cette classe au sein des échantillons de validation. Le score $\mathrm{F}$ est la moyenne harmonique de la précision et le rappel et évalue la pertinence du classificateur par classe. La précision globale détermine l'efficacité globale de l'algorithme qui est mesurée en divisant le nombre total d'échantillons correctement étiquetés par le nombre total d'échantillons testés. Le coefficient Kappa indique le degré de concordance entre les données de vérité au terrain et les valeurs prédites (Foody, 1992 ; Banko, 1998).

$$
\begin{aligned}
& P=\frac{V P}{(V P+F P)} \\
& R=\frac{V P}{(V P+F N)} \\
& \text { F Score }=2 \times\left[P^{-1}+R^{-1}\right]^{-1} \\
& P_{0}=\frac{\sum X_{i i}}{N} \\
& C_{\text {kappa }}=\frac{P_{0}-P_{e}}{1-P_{e}}
\end{aligned}
$$

où VP: Nombre de pixels correctement classés dans la classe i, FN : Nombre de pixels incorrectement non classés dans la classe $\mathrm{i}$, FP : Nombre de pixels incorrectement classés dans la classe $\mathrm{i}, \mathrm{P}$ : précision, $\mathrm{R}$ : rappel , $\mathrm{X}_{\mathrm{ii}}$ : Nombre de pixels correctement classés, $\mathrm{N}$ : Nombre total de pixels dans la matrice de confusion, $\mathrm{P}_{0}$ : Précision globale de la classification, $\mathrm{P}_{\mathrm{e}}$ : Possibilité d'accord aléatoire général.
Par ailleurs, le test de McNemar (McNemar, 1947) a été utilisé afin de déterminer s'il existait une différence statistiquement significative entre les divers scénarios de classification étudiés. Ce test est un test nonparamétrique basé sur la matrice de confusion de la classification, comme le montre l'équation [Eq. 6]. Son hypothèse nulle est qu'il n'y a pas de différence entre les précisions de classification. Une valeur de $\chi^{2}$ supérieure à 3,84 et 6,63 indique une différence significative entre deux résultats de classification respectivement à un niveau de signification $95 \%$ et $99 \%$.

$$
\chi^{2}=\frac{\left(f_{12}-f_{21}\right)^{2}}{f_{12}+f_{21}}
$$

où $f_{12}$ est le nombre de pixels correctement classés par le premier scénario, mais incorrectement classés par le deuxième scénario. $f_{21}$ est le nombre de pixels correctement classées par le deuxième scénario, mais incorrectement classées par le premier scénario.

\section{Résultats et Discussion}

\section{1 Évaluation globale}

\subsection{1. Évaluation de la précision globale}

Le tableau 3 présente les résultats du coefficient Kappa et de la précision globale pour les trois niveaux de classification et les cinq scénarios.

Tableau 3: Le coefficient Kappa et l'indice de la précision globale de la classification d'occupation du sol. (Moyenne harmonique de 10 itérations)

\begin{tabular}{clrrrrr}
\hline \multirow{2}{*}{ Nv } & Par & \multicolumn{6}{c}{ Scénario } \\
\cline { 3 - 7 } & & \multicolumn{1}{c}{ S1 } & \multicolumn{1}{c}{ S2 } & \multicolumn{1}{c}{ S2Id } & S1S2 & S1S2Id \\
\hline \multirow{2}{*}{ C } & $c_{\text {kappa }}$ & 94,20 & 98,80 & 98,90 & 99,00 & 99,00 \\
& $P_{0}$ & 94,90 & 98,90 & 99,00 & 99,00 & 99,10 \\
& $S d$ & 0,06 & 0,02 & 0,04 & 0,04 & 0,02 \\
\multirow{2}{*}{ SC } & $c_{\text {kappa }}$ & 91,30 & 98,3 & 98,30 & 98,30 & 98,50 \\
& $P_{0}$ & 91,90 & 98,40 & 98,40 & 98,50 & 98,60 \\
& $S d$ & 0,06 & 0,02 & 0,04 & 0,02 & 0,03 \\
\multirow{2}{*}{ SSC } & $c_{\text {kappa }}$ & 85,70 & 95,20 & 95,20 & 94,90 & 95,20 \\
& $P_{0}$ & 86,40 & 95,40 & 95,50 & 95,30 & 95,50 \\
& $S d$ & 0,24 & 0,12 & 0,13 & 0,11 & 0,08 \\
\hline
\end{tabular}

Nv. : Niveau, Par : Paramètre, C : Classe ; SC : Sous-classe ; SSC : Sous-sous-classe. $C_{\text {kappa }}$ : Coefficient kappa en $\% ; P_{0}$ Indice de la précision globale en \% ; Sd : Écart-type de la précision.

S1 : Sentinel-1 : S2 : Sentinel-2, S2Id : Sentinel-2 et Indices S1S2 : Sentinel-1 et Sentinel-2; S1S2Id : Sentinel-1; Sentinel2 et Indices.

Les résultats obtenus avec les différents jeux de données montrent des performances relativement similaires, comprises entre $86,4 \%$ et $99,1 \%$, avec des coefficients kappa variant entre 0,8 et 0,9 . On peut noter que les seules données radar $\mathrm{S} 1$ montrent des performances en deçà (jusqu'à $9 \%$ ) des autres combinaisons. 
De plus, les classifications faites avec des images Sentinel-2 seules présentent des indicateurs d'évaluation de précisions supérieures à celles des classifications obtenues avec des images Sentinel-1 seules avec des écarts entre $4,07 \%$ et $8,99 \%$. Ce résultat est cohérent avec les conclusions des autres études (Denize et al., 2018 ; Steinhausen et al., 2018 ; Tricht et al., 2018 ; Sun et al., 2019 ; Tavares et al., 2019) qui ont montré que les valeurs de précision globale de Sentinel-2 surpassent celle de Sentinel-1.

En outre, l'ajout des indices radiométriques aux 10 bandes Sentinel-2 ne montre pas d'améliorations significatives (<1\%). De manière attendue, les performances de classification décroissent lorsque le nombre de classes recherchées augmente en raison de l'accroissement de la complexité du problème et de la confusion entre classes. En effet, les différentes classes peuvent être très proches d'un point de vue évolution radiométrique, entraînant des confusions. De plus, un grand nombre de classes rend plus difficile la définition d'un nombre de polygones d'intérêts suffisant, qui soient statistiquement représentatifs.
Les résultats révèlent aussi une similitude en termes de précision globale lors de la combinaison des jeux de données Sentinel-1, Sentinel-2 et/ou Indices.

\subsubsection{Résultats du test McNemar}

Le tableau 4 résume la différence entre les scénarios de classification basée sur l'indicateur de la précision $\left(\mathrm{P}_{0}\right)$. Les résultats du test McNemar montrent que les valeurs $p$-value sont supérieures à $\alpha=5 \%$. Par conséquent, nous avons accepté l'hypothèse nulle. Ce qui indique que la différence entre les scénarios n'est pas significative pour les trois niveaux de classification. Néanmoins, lorsque nous appliquons ce test sur les sous-classes (une par une) l'hypothèse nulle est rejetée, ce qui signifie que la majorité des sous-classes d'occupation du sol diffèrent de manière significative en termes de précision globale. Le test McNemar a été appliqué pour chaque sousclasses au niveau des trois niveaux de classification et pour six paires de scénarios (i.e S1 vs S2, S2 vs S1S2Id, ...). Le tableau A.5 illustre les résultats de ce test par sous-classes. Pour chaque niveau, nous avons calculé la fréquence des valeurs de $\chi^{2}$ significatives et non significatives par rapport au nombre total des sous classes (figure 8).

Tableau 4: Résultats global du test McNemar de la meilleure itération.

\begin{tabular}{|c|c|c|c|c|c|c|}
\hline \multirow{2}{*}{$\begin{array}{l}\text { Paire de } \\
\text { scénarios }\end{array}$} & \multicolumn{2}{|c|}{ Classe } & \multicolumn{2}{|c|}{ Sous-classe } & \multicolumn{2}{|c|}{ Sous-sous-classe } \\
\hline & $\chi^{2}$ & $\mathrm{p}$-value & $\chi^{2}$ & $p$-value & $\chi^{2}$ & $p$-value \\
\hline S1 vs S2 & 0,0034 & 0,9535 & 0,0086 & 0,9262 & 0,0289 & 0,8650 \\
\hline $\mathrm{S} 1$ vs S1S2 & 0,0033 & 0,9545 & 0,0085 & 0,9265 & 0,0295 & 0,8637 \\
\hline S1 vs S1S2Id & 0,0032 & 0,9547 & 0,0083 & 0,9274 & 0,0286 & 0,8656 \\
\hline S2 vs S2ld & 0,0034 & 0,9537 & 0,0009 & 0,9759 & 0,0075 & 0,9310 \\
\hline $\mathrm{S} 2$ vs S1S2 & 0,0033 & 0,9545 & 0,0009 & 0,9756 & 0,0078 & 0,9296 \\
\hline S2 vs S1S2Id & 0,0004 & 0,9844 & 0,0009 & 0,9759 & 0,0074 & 0,9315 \\
\hline
\end{tabular}

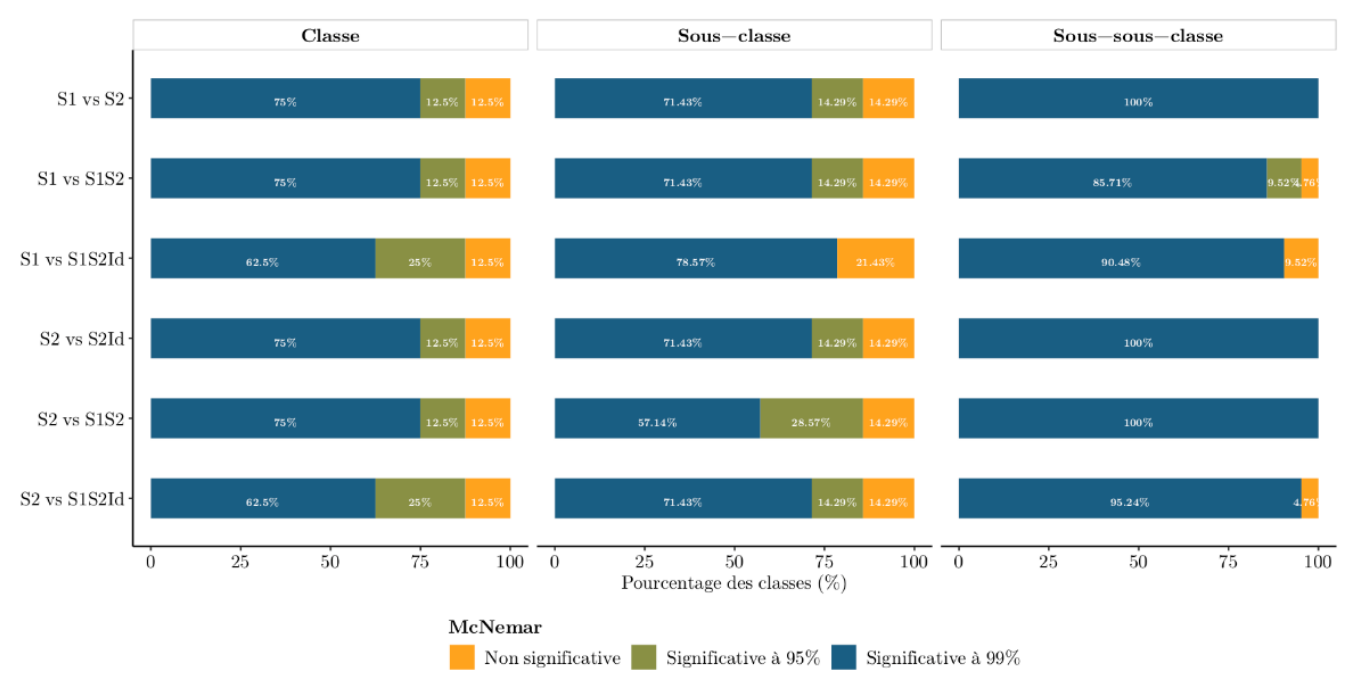

Figure 8: Le pourcentage des classes selon les résultats du test McNemar par paire de scénarios. 
Les résultats du tableau A.5 et de la figure 8 révèlent qu'en général plus de $57 \%$ des sous-classes ont des valeurs de $p$-value nettement inférieures à $\alpha=1 \%$. Ce qui implique que la différence entre les scénarios est statistiquement significative à un seuil de $1 \%$ pour toutes les paires de scénarios et les niveaux de classification, à l'exception de $9,52 \%$ à $28,57 \%$ des classes qui se sont révélées significatives au seuil de $5 \%$ tandis que $4,76 \%$ à $21,43 \%$ des classes sont non significatives. Cette différence de précision des classes est certainement due au nombre de pixels appartenant à la classe Riz, bien supérieure au nombre de pixels constituant les autres classes. Ceci souligne l'importance de choisir des classes de nombre de pixels équivalents pour ne pas biaiser les analyses statistiques résultantes. Ceci sera pris en compte dans les travaux futurs.

\subsection{Comparaison de la discrimination des classes}

Dans cet article, nous nous limitons à l'interprétation de l'indicateur F-Score qui est largement utilisé (Zhou et al., 2017 ; Sun et al., 2019). Les valeurs de F-Score des cinq scénarios de classification sont illustrées dans la figure 9.

Les valeurs moyennes de F-Score sont de $94,94 \%$, $99,10 \%, 99,01 \%$ et $99,13 \%$ pour le niveau C, de $91,94 \%$, $98,46 \%, 98,43 \%$ et $98,57 \%$ pour le niveau SC et de $86,52 \%, 95,04 \%, 95,23 \%$ et $95,24 \%$ pour le niveau SSC respectivement pour les classifications S1, S1S2, S2Id et S1S2ld. Dans l'ensemble, les performances décroissent lorsque le nombre de classes augmente.
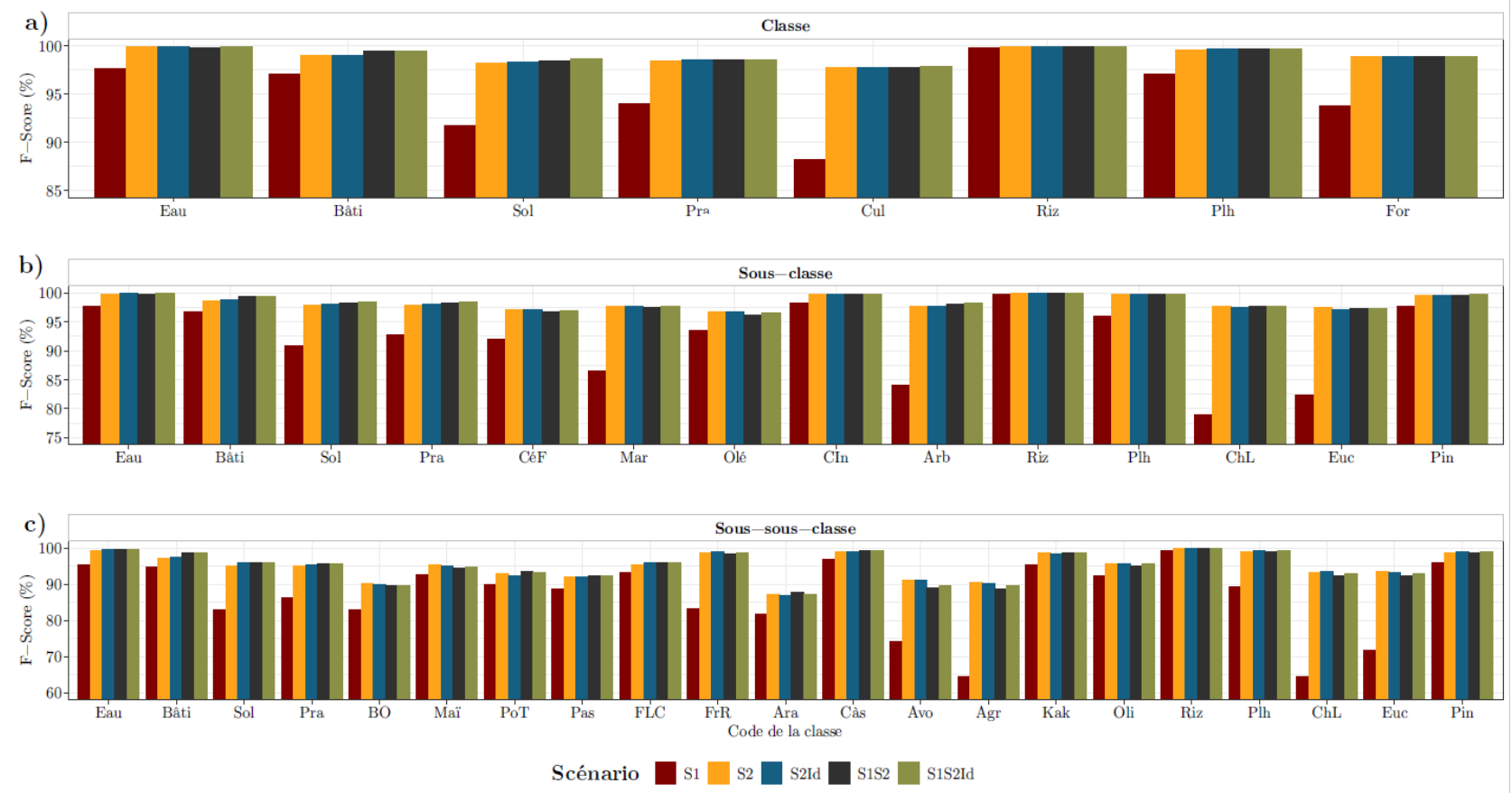

Figure 9: Résultats de l'indicateur F-Score pour chaque niveau de classification.

Ceci n'est pas surprenant puisque la complexité de la discrimination des classes augmente avec leur nombre, en raison de comportements radiométriques moins marqués entre sous-classes, et un nombre de ROI moins important. D'autre part, nous observons que pour toutes les sous-classes, les performances obtenues à partir des données optiques sont supérieures à celles obtenues avec les données radar. Ainsi des écarts moyens de $4 \%, 6 \%$, et $9 \%$ et avec des maximums de $10 \%, 19 \%$ et $29 \%$ sont observés entre S2 et $\mathrm{S} 1$, respectivement pour les niveaux C, SC, et SSC. Ces résultats ont déjà été observés dans des travaux antérieurs (Tricht et al., 2018 ; Sun et al., 2019 ; Lopes et al., 2020). De plus, la combinaison des données radar aux données optiques montre une légère amélioration $(66,67 \%$ des classes avec des écarts atteignant $1,5 \%)$ les résultats restant globalement similaires à ceux obtenus avec les données optiques seules.

On peut toutefois noter que des tendances contraires ont pu être observées dans certaines études (Sonobe et al., 2017 ; Sun et al., 2019) pour lesquelles la combinaison S1S2 a pu améliorer la discrimination de certaines classes par rapport à S2. Ainsi, une amélioration de $4,2 \%$ a été observée sur la classe de culture de haricots, et $0,7 \%$ sur le blé (Sonobe et al., 2017). On peut également remarquer que l'ajout des indices radiométriques aux canaux Sentinel-2 apporte peu d'amélioration que ce soit lors de l'utilisation des données optique seules (S2 vs S2Id) ou combinées avec le radar (S1S2 vs S1S2Id). Des résultats similaires sont obtenus avec l'indicateur de précision, les résultats les moins bons correspondant au scénario $\mathrm{S} 1$ quel que soit le niveau de la classification, à l'exception de la classe Bâti qui donne de meilleurs résultats avec le scénario S1 qu'avec le scénario S2 (avec un écart de $0,5 \%$ pour le niveau SC et $1 \%$ pour le niveau SSC).

Les cartes obtenues pour les trois niveaux et les scénarios S1, S1S2, et S1S2Id sont données en figure 10 
a)

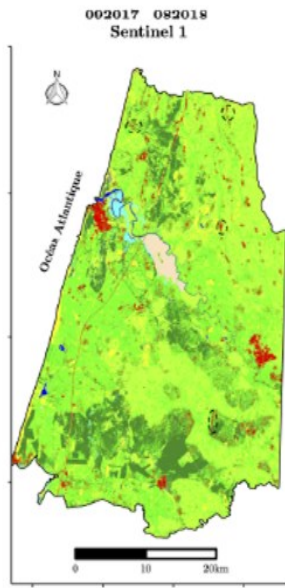

b)

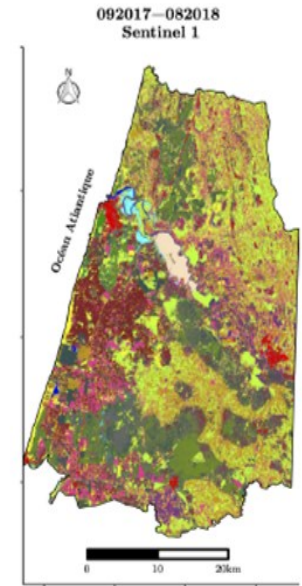

c)

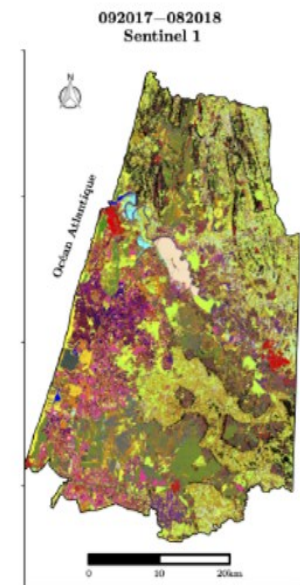

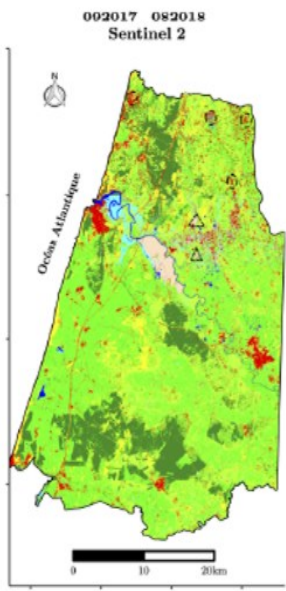

$\underset{\substack{092017-082018 \\ \text { Sentinel } 2}}{0.9}$

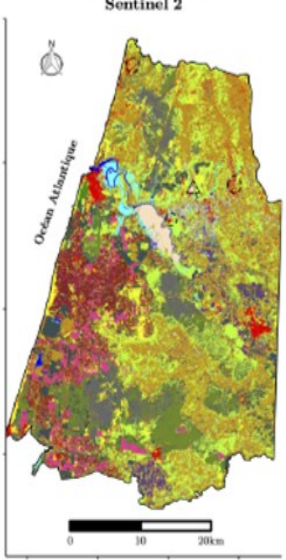

$\underset{\text { Sentinel } 2}{\text { 092018-082018 }}$

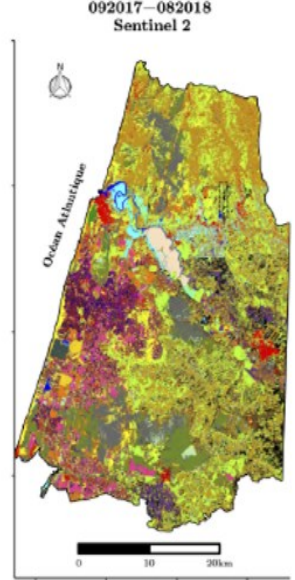

Sentinel 1, Sent 083018

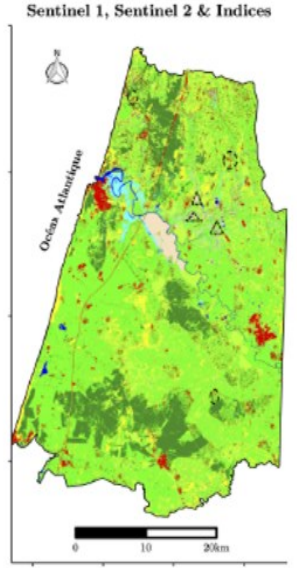

092017-082018

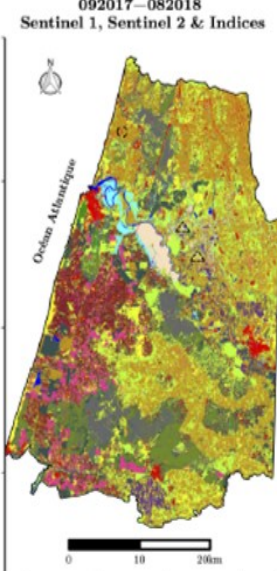

Sous-Classe

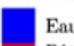

Bâtiments \&

Batiments \&

Prairie

Céréales et

fourrages

Maraichage

Oléagineux

Cultures

ndustrielles

Arboriculture

Rizière

Plantes hydrophiles

Chêne-liège

Eucalyptus

Pin

Sol nu

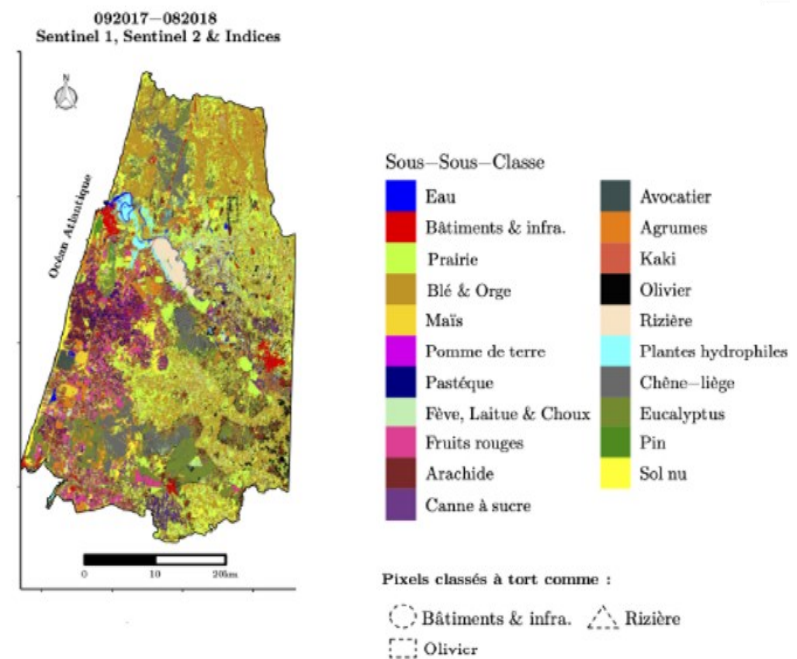

Figure 10: Classification supervisée RF par (a) classes, (b) sous-classes et (c) sous-sous-classes pour les scénarios S1, S1S2 et S1S2ld. Périmètre du Loukkos (Maroc). RF : Forêt aléatoire.

Ces cartes permettent une analyse visuelle, complémentaire de celle basée sur les statistiques présentées précédemment. Nous observons que certains pixels sont classés comme Bâti alors qu'ils devraient être classés comme Sol ou autre classe. De plus, les relevés terrain ont établi que les parcelles de riz sont réparties uniquement sur la rive gauche de l'oued Loukkos. Ce résultat est obtenu par le scénario $\mathrm{S} 1$ quel que soit le niveau de classification. Toutefois, d'autres parcelles de riz sont détectées sur la rive droite de l'oued par les autres scénarios. Au niveau sous-sous-classe, les cartes obtenues montrent que certains pixels sont classés à tort comme Oli. Cette confusion Oli et autre classe est fortement réduite par la combinaison S1S2Id. De plus, on peut voir la sensibilité des données radar au relief, qui est classé à tort comme Oli avec le scénario S1 (zone au nord de l'image). La combinaison avec les données optiques permet d'y remédier. 
Nous présentons ici les résultats en termes de superficies des classes estimées afin d'analyser la cohérence entre les différents niveaux de classification. Les résultats sont montrés figure 11 , qui représente le pourcentage de la superficie résultante pour chaque niveau de classification et par sous-classes. Les détails sont donnés dans le tableau A.6, sur la superficie estimée de chaque sous-classes, résultante de la meilleure itération, par les différents scénarios.
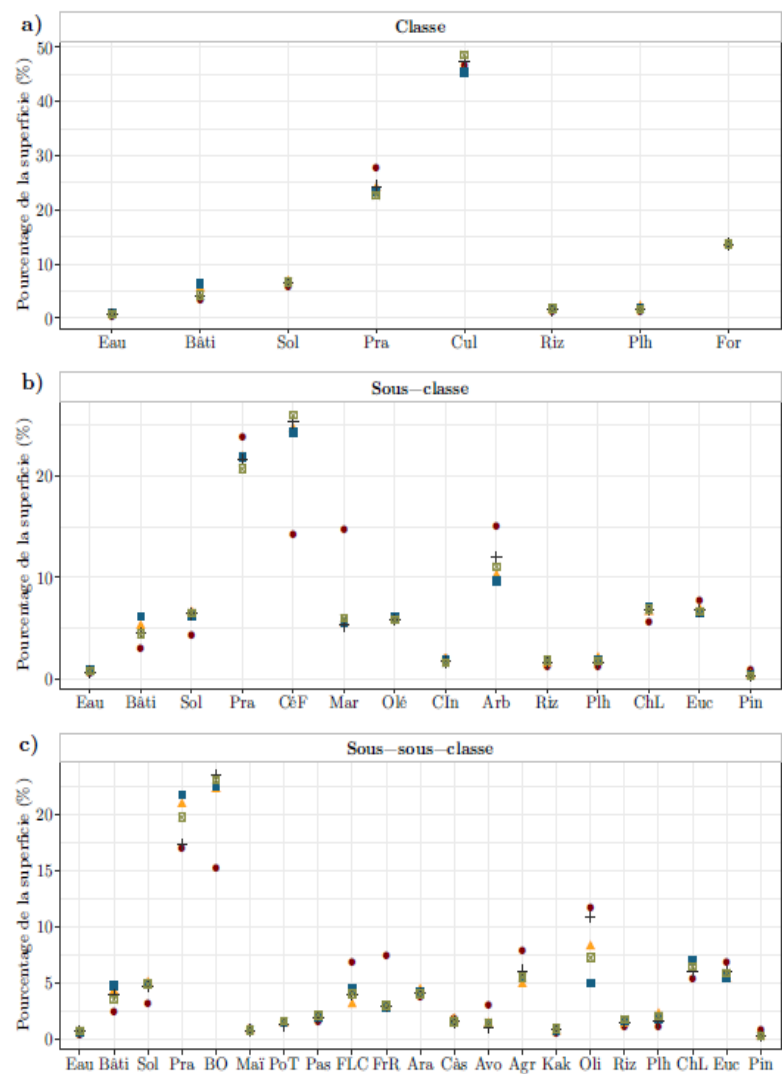

Scénario - S1 A S2 ॥ S2Id + S1S2 四 S1S2Id

Figure 11: Pourcentage de la superficie de chaque classe structurée par: (a) classes, (b) sous-classes et (c) sous-sous- classes. Périmètre du Loukkos (Maroc).

Les résultats indiquent que le pourcentage de la superficie couverte par Eau, Sol, Bâti, Pra, Riz et PIh n'a pas trop différé en passant du niveau classe au niveau sous-classe. Toutefois, elle a différé en passant au niveau sous-sous-classe avec des écarts atteignant $10,67 \%$ (S1, Pra).

Avec les informations disponibles et les résultats obtenus, il est difficile de juger si un scénario estime mieux la superficie d'une sous-classe donnée par rapport à un autre scénario. Avec plus d'informations nous pourrions comparer les superficies résultantes par chaque scénario avec celles recensées sur terrain. Des recherches futures sont prévues pour répondre à la question suivante : les valeurs élevées obtenues par les indicateurs de précision sont-elles suffisantes pour juger la bonne qualité de la classification tandis que les superficies sont sous-estimées ou surestimées?

\section{Conclusion}

Dans cette étude, nous avons étudié la complémentarité des données Sentinel-1 et Sentinel-2 pour l'estimation de l'occupation des sols dans le périmètre irrigué du Loukkos (Nord du Maroc) suivant une nomenclature hiérarchique à trois niveaux. Pour ce faire, 74 images radar Sentinel- 1 et 24 images optiques Sentinel-2 ont été utilisée sur la période d'étude allant de septembre 2017 à août 2018. Le grand nombre d'images radar permet de réduire significativement le speckle tout en conservant la résolution radiométrique en utilisant un filtre spatio-temporel.

Nous trouvons que les classifications faites à partir de données Sentinel-2 présentent des performances significativement supérieures à celles basées sur les données Sentinel-1. L'ajout d'indices radiométriques déduits des canaux optiques n'apporte pas d'amélioration par rapport à l'utilisation des 10 canaux à 10 et $20 \mathrm{~m}$ de résolutions spatiales de Sentinel-2. La combinaison des données n'améliore pas significativement les performances. Des classifications basées sur les 10 canaux de Sentinel-2 sont donc un très bon compromis en termes de volume de données à traiter et performance de classification. Elles permettent de discriminer toutes les classes avec une bonne performance (F-Score $>85 \%$ ). Ces travaux montrent le fort potentiel des données Sentinel-2, dont la période d'acquisition de 5 jours augmente significativement la possibilité d'acquisitions hors couverture nuageuse.

Les travaux futurs se concentreront sur la classification de certains types de cultures séparément tels que (blé et orge) et (fève, laitue et choux) ainsi que l'étude des profils temporels de chaque type de culture. Une comparaison entre les superficies réelles des cultures (obtenues à partir de la base de données d'historique d'état des cultures) et celles résultantes par le radar et/ou optique est recommandable.

\section{Remerciements}

Ce travail est réalisé dans le cadre du projet: PPR2/2016/79, OGI-Env, soutenu par le MENFPESRS et le CNRST. L'auteur tient à remercier le personnel de l'Office Régional de Mise en Valeur Agricole du Loukkos particulièrement $A$. Chatri et $Y$. Bensajay. II remercie aussi C. Lardeux pour la discussion scientifique et ses remarques. Un merci s'adresse à A. Acharki pour l'accompagnement en sorties de terrain. Un grand merci s'adresse également à une personne anonyme pour ses précieuses orientations et suggestions. L'auteur souhaite également remercier les réviseurs pour leurs commentaires sur cet article.

\section{Références}

Baghdadi, N., Zribi, M., 2017. Observation des surfaces continentales par télédétection IV : environnement et risques. ISTE Editions Ltd, Ch. Télédétection et mesure de la déforestation, p. 45-66.

Banko, G., novembre 1998. A review of assessing the accuracy of classifications of remotely sensed data and of methods including remote sensing data in forest inventory. Interim reportir-98-081, International Institute for Applied Systems Analysis, A-2361 Laxenburg. Austria. 
Belgiu, M., Dragut, L., apr 2016. Random forest in remote sensing: A review of applications and future directions. ISPRS Journal of Photogrammetry and Remote Sensing 114, 24-31.

Breiman, L., 2001. Machine learning. Dans: Random Forests. Vol. 45. Kluwer Academic Publishers, p. 5-32.

Congedo, L., 2016. Semi-Automatic Classification Plugin Documentation. Release 6.0.1.1.

Copernicus, 2018. Copernicus Open Data Hub. Available online: URL https://scihub.copernicus.eu.

Denize, J., Hubert-Moy, L., Betbeder, J., Corgne, S., Baudry, J., Pottier, E., dec 2018. Evaluation of using sentinel-1 and -2 time-series to identify winter land use in agricultural landscapes. Remote Sensing 11 (1), 37.

\section{ESA, E. S. A., 2015. Sentinel-2 userbook.}

Foody, G., 1992. On the compensation for chance agreement in image classification accuracy assessment. Photogrammetric Engineering and Remote Sensing 58, 1459- 1460 .

Frison, P.-L., Lardeux, C., 2018. QGIS and Application in Agriculture and Forest. Elsevier Ltd.: Oxford, UK, Ch. Vegetation Cartography and from Sentinel and Radar Images, p. 181-214.

Gao, B.-C., 1996. NDWI - a normalized difference water index for remote sensing of vegetation liquid water from space. Remote Sensing Environment 58 (3), 257266.

Haboudane, D., Millera, J. R., Patteyc, E., ZarcoTejadad, P. J., Strachane, I. B., apr 2004. Hyperspectral vegetation indices and novel algorithms for predicting green LAI of crop canopies: Modeling and validation in the context of precision agriculture. Remote Sensing of Environment 90 (3), 337-352.

Hadria, R., Duchemin, B., Baup, F., Toan, T. L., Bouvet, A., Dedieu, G., Page, M. L., jul 2009. Combined use of optical and radar satellite data for the detection of tillage and irrigation operations: Case study in central morocco. Agricultural Water Management 96 (7), 11201127.

Htitiou, A., Boudhar, A., Lebrini, Y., Hadria, R., Lionboui, H., Elmansouri, L., Tychon, B., Benabdelouahab, T., oct 2019. The performance of random forest classification based on phenological metrics derived from sentinel- 2 and landsat 8 to map crop cover in an irrigated semi-arid region. Remote Sensing in Earth Systems Sciences 2 (4), 208-224.

Huete, A., Didan, K., Miura, T., Rodriguez, E., Gao, X., Ferreira, L., 2002. Overview of the radiometric and biophysical performance of the modis vegetation indices. Remote Sensing of Environment 83, 195-213.

Höpfner, C., Scherer, D., nov 2011. Analysis of vegetation and land cover dynamics in north-western morocco during the last decade using MODIS NDVI time series data. Biogeosciences 8 (11), 3359-3373.

Jensen, J. R., 2005. Introductory Digital Image Processing: A Remote Sensing Perspective. Prentice Hall.

Lopes, M., Frison, P.-L., Crowson, M., WarrenThomas, E., Hariyadi, B., Kartika, W. D., Agus, F., Hamer, K. C., Stringer, L., Hill, J. K., Pettorelli, N., apr
2020. Improving the accuracy of land cover classification in cloud persistent areas using optical and radar satellite image time series. Methods in Ecology and Evolution 11 (4), 532-541.

McNemar, Q., June 1947. Note on the sampling error of the difference between correlated proportions or percentages. Psychometrika 12 (12), 153-157.

Merzlyaka, M. N., Gitelsonb, A. A., Chivkunovaa, O. B., Rakitinc, V. Y., 1999. Non-destructive optical detection of pigment changes during leaf senescence and fruit ripening. Physiologia Plantarum 106, 135-141.

Mohajane, M., Essahlaoui, A., Oudija, F., Hafyani, M. E., Hmaidi, A. E., Ouali, A. E., Randazzo, G., Teodoro, A. C., dec 2018. Land use/land cover (LULC) using landsat data series (MSS, TM, ETM and OLI) in azrou forest, in the central middle atlas of morocco. Environments 5 (12), 131.

OTB, 2018. OrfeoToolBox. Available online: URL https://www.orfeo-toolbox.org

Ouzemou, J.-E., Harti, A. E., Lhissou, R., Moujahid, A. E., Bouch, N., Ouazzani, R. E., Bachaoui, E. M., Ghmari, A. E., aug 2018. Crop type mapping from pansharpened landsat 8 NDVI data: A case of a highly fragmented and intensive agricultural system. Remote Sensing Applications: Society and Environment 11, 94103. 2

R Development Core Team, 2017. R: A language and environment for statistical computing. Vienna, Austria: R Foundation for Statistical Computing. URL https://www.R-project.org/.

Rijsbergen, C. J. V., 1979. Information Retrieval. Springer-Verlag New York Inc.

Rikimaru, A., Roy, P., Miyatake, S., 2002. Tropical forest cover density mapping. Tropical Ecology 43 (1), $39-47$.

Rondeaux, G., Michael Steven, M., Barett, F., 1996. Optimization of soil-adjusted vegetation indices. Remote Sens. Environ. 55, 95-107.

Rouse, J., Haas, R., Schell, J., Deering, D., 1974. Monitoring vegetation systems in the great plains with erts. Dans: S.C. Freden, E. M., (eds), M. B. (Eds.), Third Earth Resources Technology Satellite-1 Syposium. Vol. Technical Presentations. NASA SP-351, NASA, Washington, D.C., pp. 309-317.

Simonneaux, V., Duchemin, B., Helson, D., Er-Raki, S., Olioso, A., Chehbouni, A. G., jan 2008. The use of high-resolution image time series for crop classification and evapotranspiration estimate over an irrigated area in central morocco. International Journal of Remote Sensing 29 (1), 95-116.

Sonobe, R., Yamaya, Y., Tani, H., Wang, X., Kobayashi, N., ichiro Mochizuki, K., jul 2017. Assessing the suitability of data from sentinel-1a and $2 a$ for crop classification. GIScience \& Remote Sensing 54 (6), 918938.

Steinhausen, M. J., Wagner, P. D., Narasimhan, B., Waske, B., dec 2018. Combining sentinel-1 and sentinel2 data for improved land use and land cover mapping of monsoon regions. International Journal of Applied Earth Observation and Geoinformation 73, 595-604. 
Sun, C., Bian, Y., Zhou, T., Pan, J., may 2019. Using of multisource and multi-temporal remote sensing data improves crop-type mapping in the subtropical agriculture region. Sensors 19 (10), 2401.

Tavares, P., Beltrão, N., Guimarães, U., Teodoro, A., mar 2019. Integration of sentinel-1 and sentinel-2 for classification and LULC mapping in the urban area of belém, eastern brazilian amazon. Sensors 19 (5), 1140.

Tricht, K. V., Gobin, A., Gilliams, S., Piccard, I., oct 2018. Synergistic use of radar sentinel-1 and optical sentinel-2 imagery for crop mapping: A case study for belgium. Remote Sensing 10 (10), 1642. 1, 7, 8, 9 USGS, 2018. Earth Explorer. Available online: URL https://earthexplorer.usgs.gov
Veloso, A., Mermoz, S., Bouvet, A., Toan, T. L., Planells, M., Dejoux, J.-F., Ceschia, E., sep 2017. Understanding the temporal behavior of crops using sentinel-1 and sentinel-2-like data for agricultural applications. Remote Sensing of Environment 199, 415426.

Zha, Y., Gao, J., Ni, S., jan 2003. Use of normalized difference built-up index in automatically mapping urban areas from TM imagery. International Journal of Remote Sensing 24 (3), 583-594.

Zhou, T., Pan, J., Zhang, P.,Wei, S., Han, T., may 2017. Mapping winter wheat with multi-temporal SAR and optical images in an urban agricultural region. Sensors 17 (6), 1210. 
Tableau A.5: Les valeurs de khi-carré $\chi^{2}$ résultantes du test McNemar par classe.

\begin{tabular}{|c|c|c|c|c|c|c|c|}
\hline \multirow{2}{*}{ Niv. } & \multirow{2}{*}{ Code } & \multicolumn{6}{|c|}{$\gamma^{2}$} \\
\hline & & S1 vs S2 & S1 vs S1S2 & S1 vs S1S2Id & S2 vs S2Id & S2 vs S1S2 & S2 vs S1S2Id \\
\hline \multirow{8}{*}{$\begin{array}{l}\mathbb{D} \\
\mathscr{D} \\
\mathbb{O} \\
U\end{array}$} & Eau & $9,23^{*}$ & $10,83^{* *}$ & $7,43^{*}$ & $8,59^{*}$ & $10,83^{\star *}$ & $17,63^{* *}$ \\
\hline & Bâti & $106,08^{* *}$ & $157,99^{* *}$ & $137,17^{* *}$ & $106,49^{* *}$ & $157,99^{* *}$ & $5,43^{*}$ \\
\hline & Sol & $86,64^{* *}$ & $81,80^{* *}$ & $73,87^{\text {** }}$ & $92,74^{* *}$ & $81,80^{\star *}$ & $65,60^{* *}$ \\
\hline & Pra & $14,63^{\text {** }}$ & $6,57^{*}$ & $5,67^{*}$ & $15,79^{* *}$ & $6,57^{*}$ & $60,71^{* *}$ \\
\hline & Cul & $121,76^{* *}$ & $148,31^{* *}$ & $134,27^{* *}$ & $110,04^{* *}$ & $148,31^{* *}$ & $16,50^{* *}$ \\
\hline & Riz & 0,03 & 1,53 & 2,38 & 2,70 & 1,53 & 2,08 \\
\hline & Plh & $85,72^{* *}$ & $82,16^{\star *}$ & $87,23^{* *}$ & $100,37^{* *}$ & $82,16^{\star *}$ & $21,54^{* *}$ \\
\hline & For & $52,83^{* *}$ & $49,63^{* *}$ & $45,70^{* *}$ & $66,52^{* *}$ & $49,63^{* *}$ & $7,65^{\star}$ \\
\hline \multirow{14}{*}{ 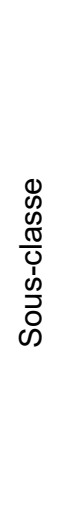 } & Eau & $9,67^{*}$ & $10,65^{*}$ & $12,07^{* *}$ & $3,89^{*}$ & 3,35 & $3,89^{*}$ \\
\hline & Bâti & $95,09^{* *}$ & $172,90^{* *}$ & $168,22^{* *}$ & $28,33^{* *}$ & $6,19^{*}$ & $28,33^{* *}$ \\
\hline & Sol & $109,38^{* *}$ & $94,15^{\star *}$ & $112,45^{\star *}$ & $103,78^{* *}$ & $45,17^{* *}$ & $103,78^{* *}$ \\
\hline & Pra & $169.93^{* *}$ & $158.41^{* *}$ & $148.18^{* *}$ & $156.52^{* *}$ & $132.00^{* *}$ & $156.52^{* *}$ \\
\hline & CéF & $390,27^{* *}$ & $426,53^{* *}$ & $403,16^{\star *}$ & $125,28^{* *}$ & $204,10^{* *}$ & $125,28^{* *}$ \\
\hline & Mar & $379,96^{\star *}$ & $395.50^{* *}$ & $385.78^{\star *}$ & $287.90^{* *}$ & $309.25^{* *}$ & $287,90^{* *}$ \\
\hline & Olé & $1002,94^{* *}$ & $1082,43^{* *}$ & $1052,72^{\star *}$ & $677,73^{* *}$ & $798,03^{* *}$ & $677,73^{* *}$ \\
\hline & Cln & 0,18 & 0,00 & 0,00 & $8,17^{*}$ & $6,57^{*}$ & $8,17^{*}$ \\
\hline & Arb & $287,72^{* *}$ & $315,76^{* *}$ & 317,50 ** & 2,80 & $7,13^{*}$ & 2,80 \\
\hline & Riz & $5,28^{*}$ & $6,62^{*}$ & 3,23 & 0,17 & 1,13 & 0,17 \\
\hline & Plh & $138,79^{* *}$ & $142,02^{* *}$ & $136,53^{* *}$ & $14,29^{* *}$ & $15,84^{* *}$ & $14,29^{* *}$ \\
\hline & $\mathrm{ChL}$ & $48,33^{* *}$ & $48,99^{* *}$ & $58,33^{* *}$ & $17,42^{* *}$ & $9,88^{*}$ & $17,42^{\text {** }}$ \\
\hline & Euc & $41,87^{* *}$ & $40,83^{\star *}$ & $47,69^{* *}$ & $68,74^{* *}$ & $54,53^{* *}$ & $68,74^{\text {** }}$ \\
\hline & Pin & 0,04 & 0,04 & 0,83 & $32,30^{* *}$ & $46,68^{* *}$ & $32,30^{* *}$ \\
\hline \multirow{21}{*}{ 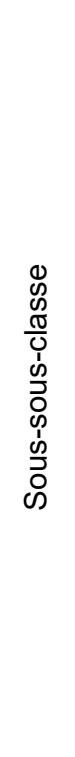 } & Eau & $15,42^{* *}$ & $51,75^{\star *}$ & $47,25^{\star *}$ & $33,36^{* *}$ & $51,08^{* *}$ & $46,58^{* *}$ \\
\hline & Bâti & $205,44^{* *}$ & $10,19^{\star}$ & 2,14 & $483,34^{* *}$ & $264,57^{* *}$ & $239,01^{* *}$ \\
\hline & Sol & $426,21^{* *}$ & $423,97^{* *}$ & $362,95^{\star *}$ & $1106,37^{* *}$ & $826,73^{* *}$ & $765,67^{* *}$ \\
\hline & Pra & $116,88^{* *}$ & $5,80^{*}$ & $15,01^{* *}$ & $329,12^{* *}$ & $66,78^{* *}$ & $85,54^{\text {** }}$ \\
\hline & $\mathrm{BO}$ & $47,40^{\star *}$ & $29,48^{\star *}$ & $44,43^{* *}$ & $240,37^{* *}$ & $123,18^{* *}$ & $146,15^{\star *}$ \\
\hline & Maї & $1908,18^{* *}$ & 1954,44 & $1705,31^{* *}$ & $3659,75^{\star *}$ & $3737,01^{* *}$ & $3484,29^{* *}$ \\
\hline & PoT & $854,50^{* *}$ & $876,38^{* *}$ & $1025,22^{* *}$ & $1971,89^{* *}$ & $1835,24^{* *}$ & $1988,26^{* *}$ \\
\hline & Pas & $788,93^{* *}$ & $1044,12^{* *}$ & $1004,70^{* *}$ & $1573,40^{* *}$ & $1918,91^{* *}$ & $1878,97^{* *}$ \\
\hline & FLC & $186,51^{\text {** }}$ & $121,65^{\star *}$ & $110,37^{* *}$ & $412,48^{* *}$ & $428,35^{\star *}$ & $418,14^{* *}$ \\
\hline & FrR & $1125,59^{* *}$ & $1230,59^{* *}$ & $1524,91^{\text {** }}$ & $2689,64^{\star *}$ & $2458,90^{* *}$ & $2761,91^{\text {** }}$ \\
\hline & Ara & $772,82^{* *}$ & $952,50^{* *}$ & $512,03^{* *}$ & $1128,95^{\star *}$ & $1685,92^{* *}$ & $1224,94^{* *}$ \\
\hline & Càs & $51,25^{\star *}$ & $311,36^{* *}$ & $170,50^{* *}$ & $271,80^{\text {** }}$ & $350,36^{* *}$ & $236,05^{\text {** }}$ \\
\hline & Avo & $877,60^{* *}$ & $797,04^{* *}$ & $893,65^{\star *}$ & $2350,84^{* *}$ & $1910,62^{* *}$ & $2024,10^{\text {** }}$ \\
\hline & Agr & $316,05^{\star *}$ & $399,56^{* *}$ & $479,57^{* *}$ & $756,05^{\star *}$ & $758,79^{* *}$ & $838,97^{* *}$ \\
\hline & Kak & $248,40^{* *}$ & $221,47^{* *}$ & $258,67^{* *}$ & $718,38^{* *}$ & $587,47^{* *}$ & $627,34^{* *}$ \\
\hline & Oli & $1393,32^{* *}$ & $2259,08^{* *}$ & $2057,07^{* *}$ & $2962,54^{\star *}$ & $3686,68^{* *}$ & $3484,57^{* *}$ \\
\hline & $\mathrm{Riz}$ & $44,58^{* *}$ & $17,39^{\star *}$ & $17,13^{\text {** }}$ & $75,36^{* *}$ & $78,13^{\star *}$ & $81,26^{* *}$ \\
\hline & Plh & $69,51^{\text {** }}$ & $31,36^{\star *}$ & 2,13 & $26,32^{\text {** }}$ & $17,09^{* *}$ & 0,49 \\
\hline & $\mathrm{ChL}$ & $2121,87^{\text {** }}$ & $1705,05^{* *}$ & $2005,55^{\star *}$ & $4589,98^{\star *}$ & $4069,78^{\star *}$ & $4436,14^{* *}$ \\
\hline & Euc & $2784,49^{* *}$ & $2316,05^{* *}$ & $2669,90^{\star *}$ & $5181,34^{* *}$ & $5453,83^{* *}$ & $5861,33^{* *}$ \\
\hline & Pin & $188,45^{\text {** }}$ & $225,33^{* *}$ & $159,20^{* *}$ & $456,47^{* *}$ & $525,88^{* *}$ & $451,23^{* *}$ \\
\hline
\end{tabular}

${ }^{* *}$ : statistiquement significative au niveau de signification de $1 \%,{ }^{*}$ : statistiquement significative au niveau de signification de $5 \%$, les autres sont statistiquement non significatives

Bâti : Bâtiments \& infra ; Sol : Sol nu ; Pra : Prairie ; Cul : Cultures ; Riz : Rizière ; Plh : Plantes hydrophiles ; For : Forêt. ; CéF : Céréales et fourrages ; Mar : Maraîchage ; Olé : Oléagineux; Cln : Cultures industrielles ; Arb : Arboriculture ; ChL : Chêne-liège ; Euc : Eucalyptus, Pin. ; BO : Blé \& Orge ; Maï : Maïs ; PoT : Pomme de terre ; Pas : Pastèque ; FLC : Fève, Laitue \& Choux; FrR : Fruits rouges ; Ara : Arachide ; Càs : Canne à sucre ; Avo : Avocatier; Agr : Agrumes; Kak: Kaki ; Oli : Olivier 
Tableau A.6: Superficie en $\mathrm{Km}^{2}$ et en \% de la meilleure itération pour les trois niveaux de classification

\begin{tabular}{|c|c|c|c|c|c|c|c|c|c|c|c|}
\hline \multirow{3}{*}{ Niv. } & \multirow{3}{*}{ Code } & & & & & \multicolumn{6}{|c|}{ Scénario } \\
\hline & & \multicolumn{2}{|c|}{ S1 } & \multicolumn{2}{|c|}{ S2 } & \multicolumn{2}{|c|}{ S2Id } & \multicolumn{2}{|c|}{ S1S2 } & \multicolumn{2}{|c|}{ S1S2Id } \\
\hline & & $\mathrm{km}^{2}$ & $\%$ & $\mathrm{~km}^{2}$ & $\%$ & $\mathrm{~km}^{2}$ & $\%$ & $\mathrm{~km}^{2}$ & $\%$ & $\mathrm{~km}^{2}$ & $\%$ \\
\hline \multirow{8}{*}{$\begin{array}{l}0 \\
\infty \\
\infty \\
\infty \\
\frac{\pi}{U}\end{array}$} & Eau & 8,10 & 0,48 & 13,04 & 0,77 & 13,75 & 0,81 & 11,15 & 0,66 & 13,19 & 0,78 \\
\hline & Bâti & 55,84 & 3,30 & 93,60 & 5,53 & 109,15 & 6,45 & 70,45 & 4,16 & 70,34 & 4,16 \\
\hline & Sol & $\overline{97,51}$ & 5,76 & 114,70 & 6,78 & 114,20 & 6,75 & 111,26 & 6,57 & 112,73 & 6,66 \\
\hline & Pra & 467,71 & 27,64 & 407,06 & 24,05 & 395,22 & 23,35 & 411,68 & 24,33 & 383,77 & 22,68 \\
\hline & Cul & 789,12 & 46,63 & 767,30 & 45,34 & 768,09 & 45,39 & 803,31 & 47,47 & $\overline{822,27}$ & 48,59 \\
\hline & Riz & 19,35 & 1,14 & 26,87 & 1,59 & 32,23 & 1,90 & 26,25 & 1,55 & 29,27 & 1,73 \\
\hline & Plh & 20,48 & 1,21 & 36,77 & 2,17 & 29,71 & 1,76 & 27,38 & 1,62 & 28,34 & 1,67 \\
\hline & For & $\overline{234,21}$ & 13,84 & 232,98 & 13,77 & $\underline{229,95}$ & 13,59 & 230,84 & 13,64 & 232,40 & 13,73 \\
\hline \multirow{14}{*}{$\begin{array}{l}0 \\
\mathscr{D} \\
D \\
\mathbb{0} \\
0 \\
\dot{1} \\
\mathscr{D} \\
0 \\
\infty\end{array}$} & Eau & $\underline{7,87}$ & 0,47 & 13,05 & 0,77 & 14,55 & 0,86 & 11,08 & 0,65 & 12,43 & 0,73 \\
\hline & Bâti & 49,71 & 2,94 & 88,25 & 5,22 & 104,26 & 6,16 & 75,81 & 4,48 & 74,69 & 4,41 \\
\hline & Sol & 72,60 & 4,29 & 111,02 & 6,56 & 104,73 & 6,19 & 108,35 & 6,40 & 109,66 & 6,48 \\
\hline & Pra & $\overline{405,37}$ & 23,95 & 370,55 & 21,90 & 370,07 & 21,87 & 365,80 & 21,62 & $\underline{350,58}$ & 20,72 \\
\hline & CéF & 241,09 & 14,25 & 417,96 & 24,70 & 412,49 & 24,37 & 428,95 & 25,35 & $\overline{440,38}$ & 26,02 \\
\hline & Mar & $\overline{249,04}$ & 14,72 & 92,70 & 5,48 & 94,01 & 5,55 & 89,06 & 5,26 & 100,67 & 5,95 \\
\hline & Olé & 98,57 & 5,82 & 101,20 & 5,98 & 103,03 & 6,09 & 97,64 & 5,77 & 98,44 & 5,82 \\
\hline & $\mathrm{Cln}$ & 35,22 & 2,08 & 32,12 & 1,90 & 30,09 & 1,78 & 27,82 & 1,64 & 26,08 & 1,54 \\
\hline & Arb & 254,61 & 15,05 & 172,58 & 10,20 & 162,86 & 9,62 & 202,17 & 11,95 & 186,85 & 11,04 \\
\hline & Riz & 18,92 & 1,12 & 25,61 & 1,51 & 30,79 & 1,82 & 25,67 & 1,52 & 30,19 & 1,78 \\
\hline & Plh & 19,77 & 1,17 & 34,66 & 2,05 & 31,98 & 1,89 & 26,83 & 1,59 & 29,33 & 1,73 \\
\hline & ChL & $\overline{94,12}$ & 5,56 & 111,40 & 6,58 & 118,52 & 7,00 & 114,69 & 6,78 & 116,03 & 6,86 \\
\hline & Euc & $\overline{130,91}$ & 7,74 & 115,66 & 6,83 & 108,83 & 6,43 & 114,08 & 6,74 & 112,25 & 6,63 \\
\hline & Pin & 14,52 & 0,86 & 5,55 & 0,33 & 6,13 & 0,36 & $\underline{4,38}$ & 0,26 & 4,74 & 0,28 \\
\hline \multirow{21}{*}{ 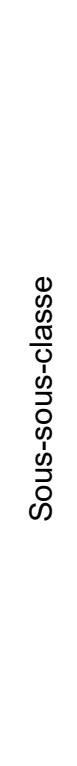 } & Eau & $\underline{6,54}$ & 0,39 & 9,85 & 0,58 & 10,89 & 0,64 & 10,27 & 0,61 & 11,72 & 0,69 \\
\hline & Bâti & 39,78 & 2,35 & 71,90 & 4,25 & 80,00 & 4,73 & 66,01 & 3,90 & 58,95 & 3,48 \\
\hline & Sol & 52,64 & 3,11 & 84,80 & 5,01 & 81,08 & 4,79 & 78,55 & 4,64 & 81,94 & 4,84 \\
\hline & Pra & 287,09 & 16,96 & 354,37 & 20,94 & 368,14 & 21,75 & 293,81 & 17,36 & 334,40 & 19,76 \\
\hline & $\mathrm{BO}$ & 256,76 & 15,17 & 376,87 & 22,27 & 381,11 & 22,52 & 397,56 & 23,49 & 391,00 & 23,10 \\
\hline & Maï & 16,28 & 0,96 & 11,65 & 0,69 & 11,17 & 0,66 & 12,45 & 0,74 & 13,00 & 0,77 \\
\hline & РoT & 24,47 & 1,45 & 22,76 & 1,35 & 24,70 & 1,46 & 20,46 & 1,21 & 26,10 & 1,54 \\
\hline & Pas & $\underline{25,70}$ & 1,52 & 33,73 & 1,99 & 32,78 & 1,94 & $\overline{30,78}$ & 1,82 & 35,82 & 2,12 \\
\hline & FLC & $\overline{115,67}$ & 6,83 & $\underline{51,86}$ & 3,06 & 74,84 & 4,42 & 66,82 & 3,95 & 67,93 & 4,01 \\
\hline & FrR & 125,53 & 7,42 & 47,93 & 2,83 & 48,14 & 2,84 & 48,58 & 2,87 & 50,25 & 2,97 \\
\hline & Ara & 63,27 & 3,74 & 74,14 & 4,38 & 70,67 & 4,18 & 69,45 & 4,10 & 67,00 & 3,96 \\
\hline & Càs & 30,73 & 1,82 & 28,93 & 1,71 & 24,70 & 1,46 & 25,57 & 1,51 & 25,31 & 1,50 \\
\hline & Avo & 50,94 & 3,01 & 22,62 & 1,34 & $\overline{23,85}$ & 1,41 & $\underline{15,93}$ & 0,94 & 23,92 & 1,41 \\
\hline & Agr & 134,19 & 7,93 & $\underline{82,08}$ & 4,85 & 90,94 & 5,37 & $\overline{102,22}$ & 6,04 & 92,67 & 5,48 \\
\hline & Kak & $\underline{7,89}$ & 0,47 & 10,82 & 0,64 & 13,12 & 0,78 & 14,33 & 0,85 & 15,40 & 0,91 \\
\hline & Oli & $\overline{198,60}$ & 11,74 & 139,33 & 8,23 & 83,57 & 4,94 & 183,50 & 10,84 & 121,95 & 7,21 \\
\hline & $\mathrm{Riz}$ & 19,09 & 1,13 & 22,77 & 1,35 & 28,23 & 1,67 & 23,67 & 1,40 & 29,09 & 1,72 \\
\hline & Plh & 17,86 & 1,06 & 37,43 & 2,21 & 30,28 & 1,79 & 25,31 & 1,50 & 34,32 & 2,03 \\
\hline & $\mathrm{ChL}$ & 89,75 & 5,30 & 113,97 & 6,73 & 117,66 & 6,95 & 101,79 & 6,01 & 108,97 & 6,44 \\
\hline & Euc & $\overline{115,38}$ & 6,82 & 90,67 & 5,36 & 92,26 & 5,45 & 101,56 & 6,00 & 98,97 & 5,85 \\
\hline & Pin & 14,15 & 0,84 & 3,82 & 0,23 & 4,19 & 0,25 & 3,70 & 0,22 & 3,61 & 0,21 \\
\hline
\end{tabular}

$\underline{\mathrm{V}}$ : Valeur minimale. $\mathbf{V}$ : Valeur maximale.

Bâti : Bâtiments \& infra ; Sol : Sol nu ; Pra : Prairie ; Cul : Cultures ; Riz : Rizière ; Plh : Plantes hydrophiles ; For : Forêt. ; CéF : Céréales et fourrages ;

Mar : Maraîchage ; Olé : Oléagineux; Cln : Cultures industrielles ; Arb : Arboriculture ; ChL : Chêne-liège ; Euc : Eucalyptus, Pin. ; BO : Blé \& Orge ;

Maï : Maïs ; PoT : Pomme de terre ; Pas : Pastèque ; FLC : Fève, Laitue \& Choux ; FrR : Fruits rouges ; Ara : Arachide ; Càs : Canne à sucre ;

Avo : Avocatier ; Agr : Agrumes; Kak : Kaki ; Oli : Olivi 